\title{
The Users, Uses, and Value of Landsat and Other Moderate-Resolution Satellite Imagery in the United States-Executive Report
}

By Holly M. Miller, Natalie R. Sexton, Lynne Koontz, John Loomis, Stephen R. Koontz, and Caroline Hermans

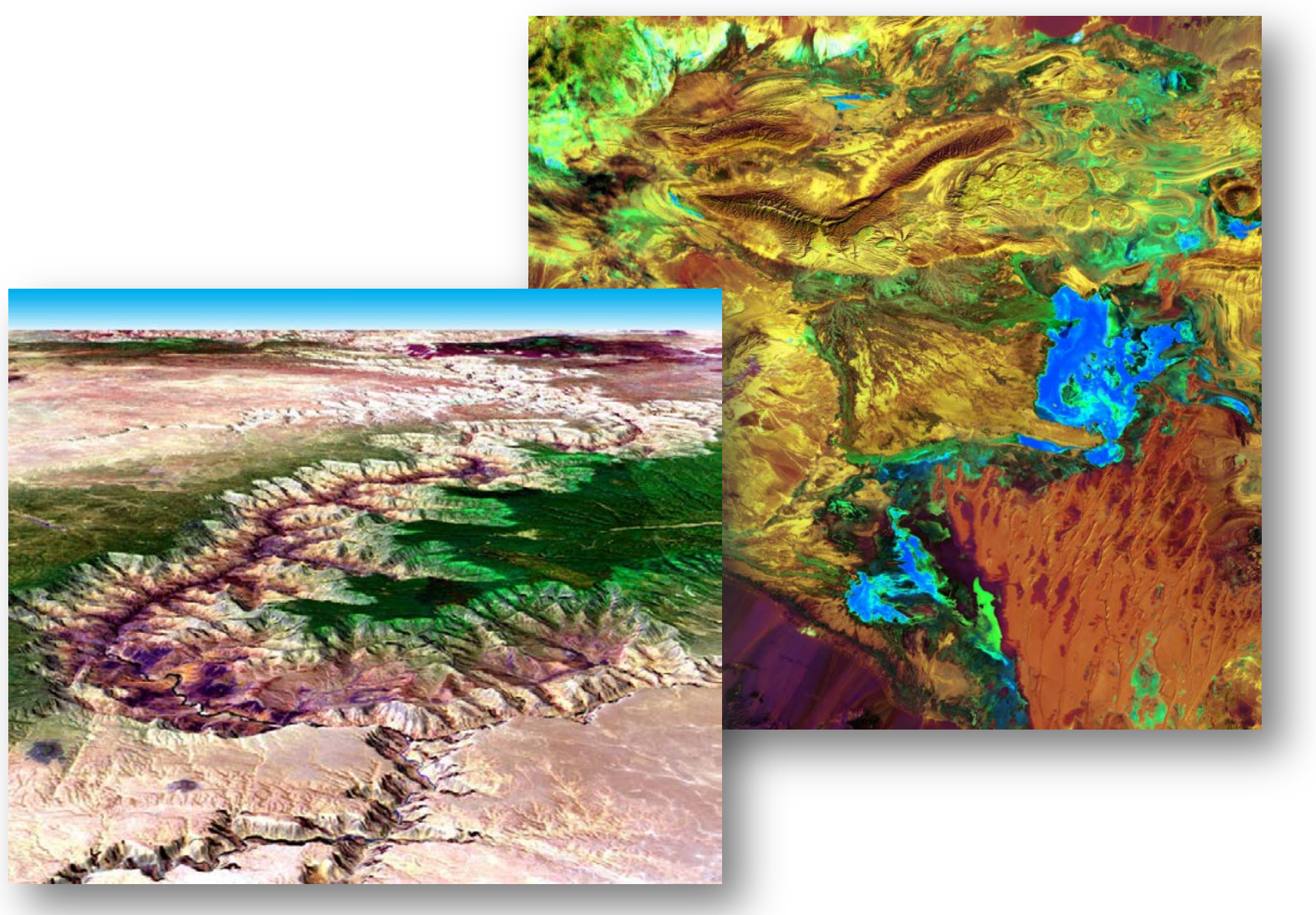

Open-File Report 2011-1031

U.S. Department of the Interior

U.S. Geological Survey 


\section{U.S. Department of the Interior KEN SALAZAR, Secretary}

\section{U.S. Geological Survey Marcia K. McNutt, Director}

U.S. Geological Survey, Reston, Virginia 2011

For product and ordering information: World Wide Web: http://www.usgs.gov/pubprod Telephone: 1-888-ASK-USGS

For more information on the USGS-the Federal source for science about the Earth, its natural and living resources, natural hazards, and the environment: World Wide Web: http://www.usgs.gov

Telephone: 1-888-ASK-USGS

Suggested citation:

Miller, H.M., Sexton, N.R., Koontz, Lynne, Loomis, John, Koontz, S.R., and Hermans, Caroline, 2011, The users, uses, and value of Landsat and other moderate-resolution satellite imagery in the United States-Executive report: U.S. Geological Survey Open-File Report 2011-1031, 43 p.

Any use of trade, product, or firm names is for descriptive purposes only and does not imply endorsement by the U.S. Government.

Although this report is in the public domain, permission must be secured from the individual copyright owners to reproduce any copyrighted material contained within this report. 


\section{Contents}

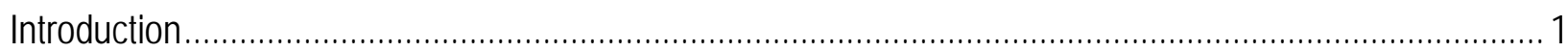

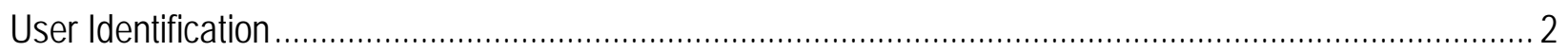

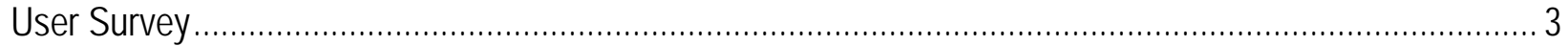

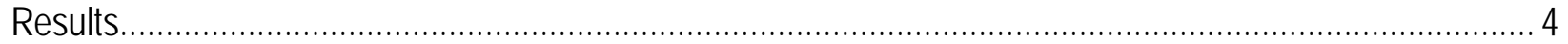

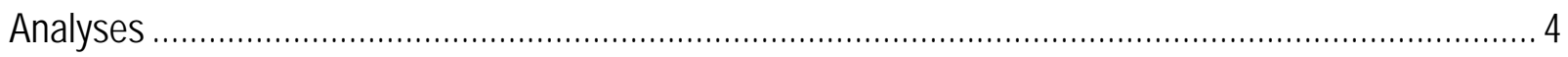

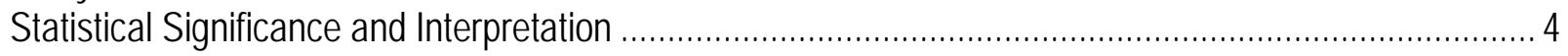

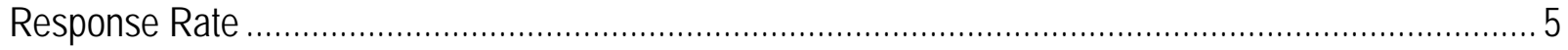

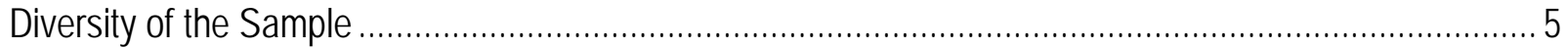

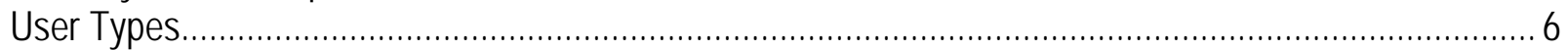

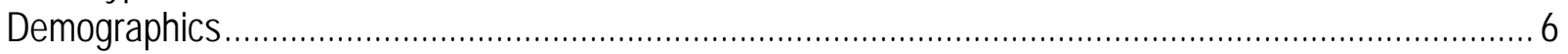

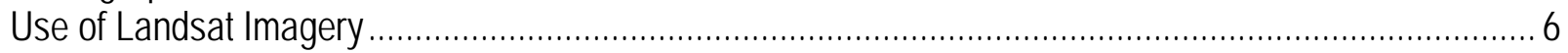

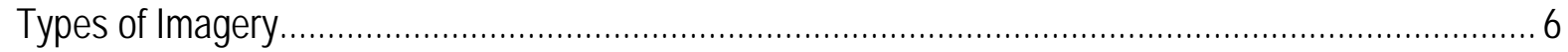

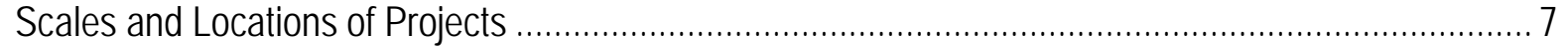

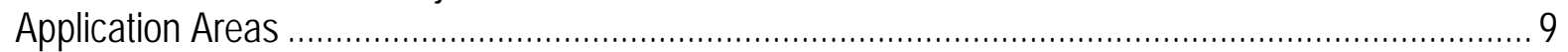

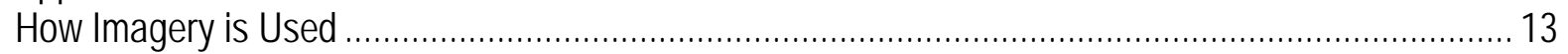

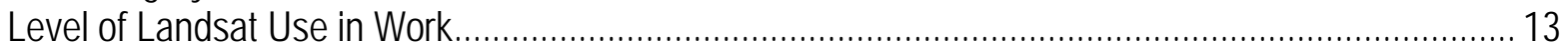

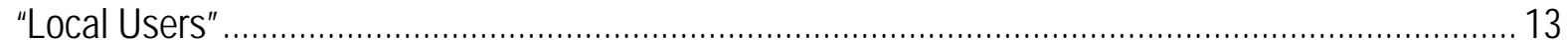

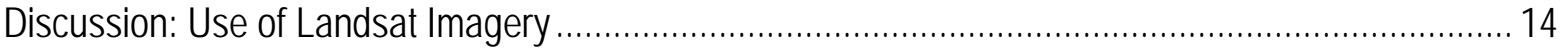

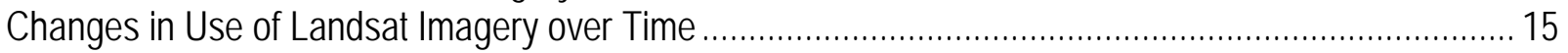

Discussion: Change in Use of Landsat Over Time .................................................................. 18

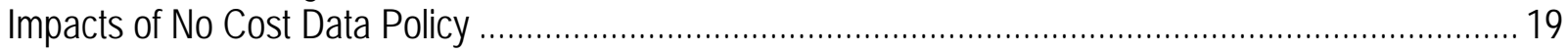

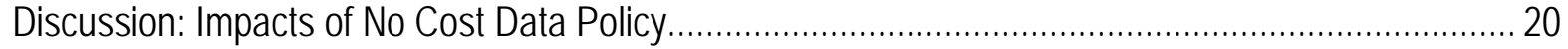

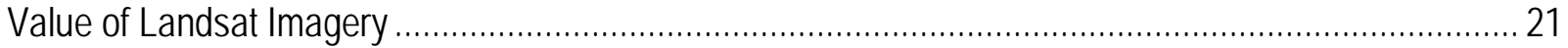

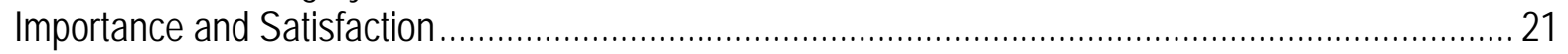

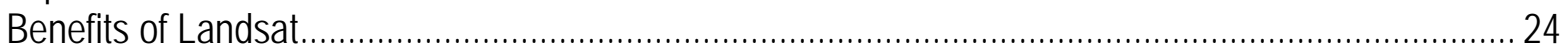

If Landsat Imagery Was No Longer Available......................................................................... 25

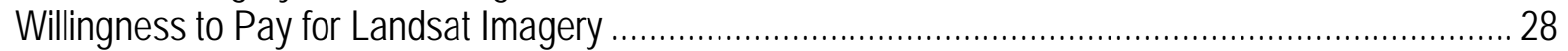

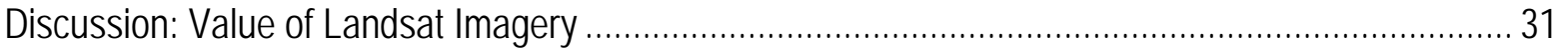

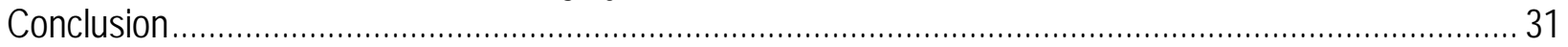

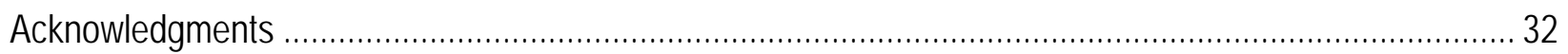

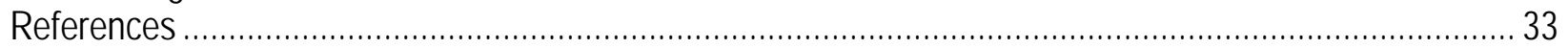

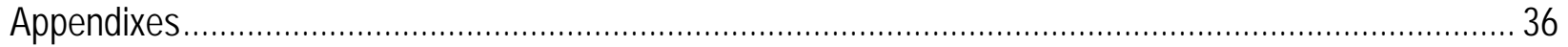

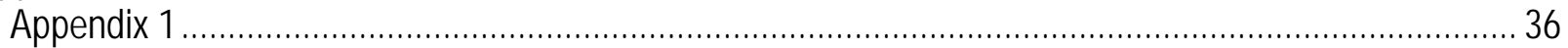

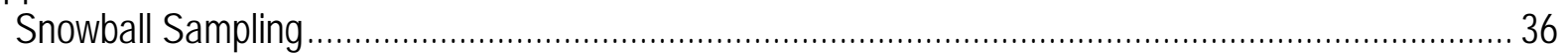

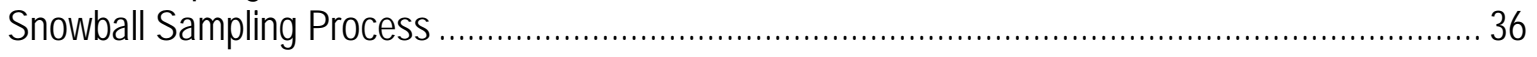

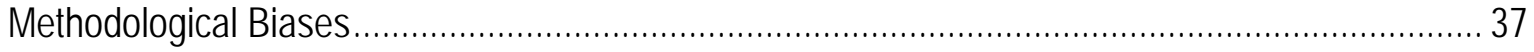

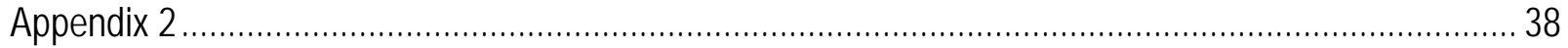

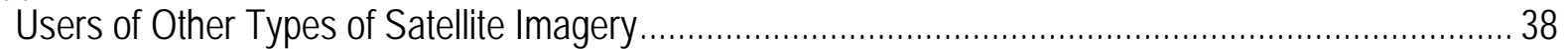

Current Users of Other Types of MRI ........................................................................... 38

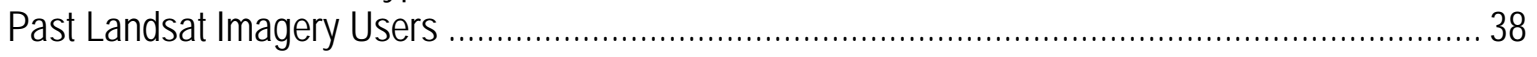

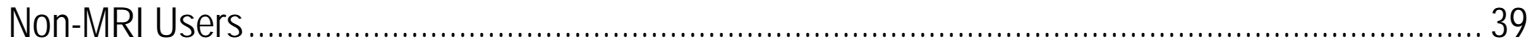

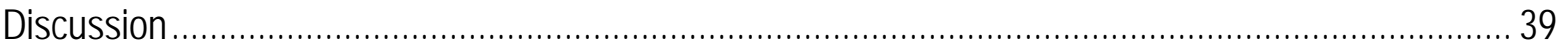




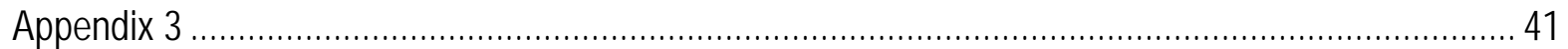

Double-Bounded Willingness-To-Pay Analysis ....................................................................... 41

Single-Bounded vs. Double-Bounded WTP Analyses .............................................................. 42

\section{Figures}

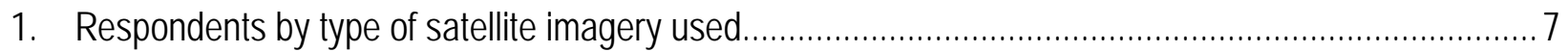

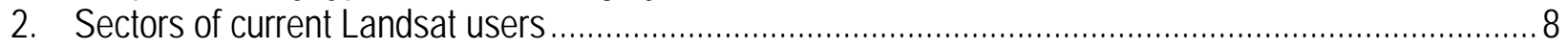

3. Locations of projects using Landsat imagery in year previous to survey ......................................... 9

4. Scales of projects using Landsat imagery in year previous to survey...............................................10

5. Applications of Landsat imagery in projects conducted in year previous to survey .............................12

6. General use of moderate-resolution imagery among current Landsat users .....................................14

7. Level of use of Landsat imagery among current Landsat users(as measured by the percentage of respondents' work that used Landsat imagery in year previous to survey)............................................. 15

8. Actual and predicted changes in use of Landsat imagery in past 10 years and next 5 years among current Landsat users.......................................................................................................... 16

9. Predicted future use of Landsat and other moderate-resolution imagery among past Landsat

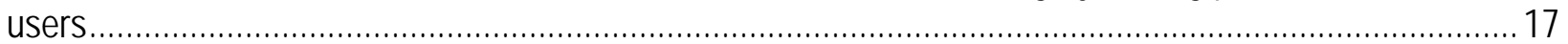

10. Reactions to Landsat 7 imagery with SLC-off among current Landsat users who indicated their Landsat use had decreased over the past 10 years ......................................................................... 18

11. Sources used by current Landsat users to obtain Landsat imagery before and after the imagery became available at no cost from USGS EROS (2008 versus 2009) ................................................. 19 12. Current Landsat users' patterns of acquisitions of Landsat imagery from USGS EROS before

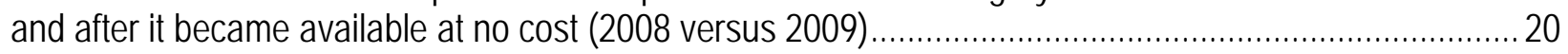

13. Importance of Landsat imagery to the work of current Landsat users .............................................. 22

14. Mean importance of and mean satisfaction with certain attributes of Landsat imagery among current Landsat users ........................................................................................................... 23

15. Preferred imagery versus imagery most likely to be acquired within budget constraints among current Landsat users who would substitute for Landsat imagery if it was no longer available....................26 16. Preferred imagery versus imagery most likely to be acquired within budget constraints among current Landsat users who would substitute different imagery based on budget constraints if Landsat imagery was no longer available ............................................................................................... 27

17. Single-bounded demand curve of willingness to pay for imagery to replace Landsat imagery among current Landsat users ................................................................................................... 29

18. Single-bounded logit model results for willingness to pay for imagery to replace Landsat imagery

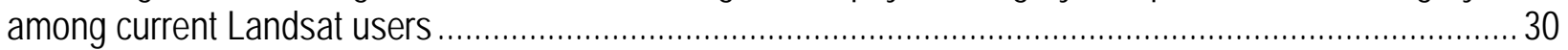
19. Single- and double-bounded demand curves of willingness to pay for imagery to replace Landsat. 


\section{Tables}

1. Mean percentage of imagery used in the year previous to the survey among current Landsat users who used a mix of moderate-resolution imagery.

2. Applications of Landsat imagery among current Landsat users .................................................... 11

3. Acquisitions of Landsat imagery before and after it became available at no cost (2008 versus 2009) by current Landsat users ............................................................................................... 21

4. Percentages of current Landsat users who would take each of three actions if Landsat imagery was no longer available 25

5. Percentages of current Landsat users who would use each of three types of information as substitutes for Landsat imagery if it was no longer available. 26 


\section{The Users, Uses, and Value of Landsat and Other Moderate-Resolution Satellite Imagery in the United States-Executive Report}

By Holly M. Miller, ${ }^{1}$ Natalie R. Sexton, ${ }^{1}$ Lynne Koontz, ${ }^{1}$ John Loomis, ${ }^{2}$ Stephen R. Koontz, ${ }^{2}$ and Caroline Hermans ${ }^{3}$

\section{Introduction}

A variety of satellites provide remotely sensed images of the earth at different pixel resolutions, generally categorized as high, moderate, or low resolution. According to the 2007 Future of Land Imaging Interagency Working Group report (FLIIWG, 2007), moderateresolution imagery (MRI) is defined as imagery that:

- covers relatively large geographic areas per scene $\left(>60 \mathrm{~km}^{2}\right)$,

- has a spatial resolution between 5 and 120 meters per image pixel,

- is characterized by repetitive coverage, and

- includes measurements from several portions of the electromagnetic spectrum.

MRI is provided by an assortment of satellites operated by governments and private businesses around the world. A main source of MRI is the Landsat satellites operated by the U.S. Geological Survey (USGS), which receives, processes, distributes, and archives Landsat imagery at the Earth Resources Observation and Science (EROS) Center. Currently, the Landsat 5 and Landsat 7 satellites are collecting imagery while in earth orbit. They provide imagery that is unique among the variety of MRI available today for three main reasons. First, the archive of imagery extends back to 1972, allowing for broad-area analyses over several decades. Second, the imagery is and has been collected globally on a regular basis, providing repeat coverage of remote areas that other satellites do not offer. Third, the imagery is currently available at no cost and with no user restrictions to those requesting images from EROS.

In the past decade, many changes have occurred both with the Landsat satellites themselves and with the provision of the imagery. In 1999, Landsat 7 was launched and provided four years of high-quality data before it sustained a critical technical malfunction in May 2003. The imaging instrument's scan-line corrector failed and this limitation (commonly referred to as SLC-off) has since reduced the quality and usability of Landsat 7 imagery. The Landsat Data Continuity Mission (LDCM or Landsat 8) is scheduled to be launched in December 2012 and will become the primary source of Landsat imagery once operational. While there are indications that both Landsat 5 and 7 will continue to operate beyond 2012, either could cease to operate before the launch of LDCM, creating a gap in the provision of imagery. Additionally, the entire

\footnotetext{
${ }^{1}$ Policy Analysis and Science Assistance Branch, U.S. Geological Survey, Fort Collins, Colo.

${ }^{2}$ Department of Agricultural and Resource Economics, Colorado State University, Fort Collins, Colo.

${ }^{3}$ The City University of New York, New York, N.Y.
} 
archive of Landsat imagery, including all new acquisitions, became available at no cost at the beginning of 2009, causing more than a 50-fold annual increase in the number of scenes downloaded from EROS with more than four million scenes downloaded by users before the end of 2010.

Moderate-resolution imagery, such as Landsat, provides unique spatial information for use by many people both within and outside of the United States (U.S.). However, exactly who these users are, how they use the imagery, and the value and benefits derived from the information are, to a large extent, unknown. The last comprehensive evaluations were completed more than 35 years ago and attempted to project the conceivable economic benefits of a continued Landsat program (ECON, Inc., 1974; EarthSat, 1974). Much has changed since that time-not only with the characteristics of remotely sensed data but the applications of the imagery. More recently, a small number of studies have surveyed limited groups of users of MRI (for example, American Society for Photogrammetry and Remote Sensing, 2006; EROS, 2007; National States Geographic Information Council, 2006; Stoney, Fletcher, and Lowe, 2001). While these surveys have added to the body of knowledge regarding opinions on the attributes of various sensors, they have not been comprehensive in nature. Our objectives for this study were to: 1) identify and classify U.S.-based users of this imagery; 2) better understand how and why MRI, and specifically Landsat, is being used; and 3) qualitatively and quantitatively measure the value and societal benefits of MRI (focusing on Landsat specifically). To reach these objectives, we conducted a study of U.S.-based MRI users from 2008 through 2010 in two parts: 1) a user identification and 2) a user survey.

\section{User Identification}

We defined users for this study as those who use MRI in any capacity in their work, as opposed to people who use MRI for personal purposes (for example, many Google Earth users). Studying these users is challenging because the extent of the population is unknown and thus, a representative sample cannot be obtained from the entire population. The membership lists of various remote sensing and geographic information systems (GIS) professional organizations could be used, but not every user can be assumed to be a member of such organizations. The lists of users from imagery providers, such as USGS EROS, which distributes Landsat, also would not include all the users in the U.S. One reason for this is that data procured by one user is often re-distributed to other users, particularly when licensing or distribution restrictions do not exist, as is the case with Landsat. Taking a random sample of the entire population of U.S. residents would not produce usable results, given that the percentage of people in the U.S. who may be classified as users of MRI is most likely quite low, making this a rare population as well. Because traditional random sample selection methods were not adequate or appropriate for this unknown population, we used a purposive sampling procedure, explained below.

For this survey, the sample was identified in a two-step process. First, an extensive web search was performed in the summer of 2008 to identify potential users of MRI in the U.S. The search was conducted by state and was based on an extensive list of keywords, including remote sensing, moderate-resolution satellite imagery, and Landsat, along with a variety of application areas and sectors where use may occur. This search yielded the email addresses of more than 20,000 potential users from across the U.S. Subsequently, snowball sampling was used to confirm the use of MRI and find additional users. Snowball sampling is a purposive (nonprobability) sampling method based on the existence of social networks (see Appendix 1 for a more comprehensive overview of snowball sampling). In any population, it can be assumed there 
are connections between the members, particularly when that population is specialized in any way, as is the population of MRI users. Using satellite imagery requires a certain level of technical knowledge and there are numerous organizations and communities that exist to facilitate the use of satellite imagery. To take advantage of these social networks, each potential user identified during the initial web search was contacted via email, asked to (1) participate in the study, (2) confirm his/her use of MRI and (3) provide the contact information for up to three other users. These newly identified potential users were contacted and asked to provide the same information. This process repeated until less than 100 new users were identified in a "wave." Snowball sampling is intended to increase the size of a sample with each wave of new participants, though well-known people within a population tend to be recommended multiple times.

By the conclusion of the snowball sampling, more than 4,000 more email addresses had been added to the list of potential users from the web search, totaling approximately 25,400 unique email addresses, of which more than 3,500 were undeliverable. Of the almost 22,000 individuals remaining, 5,229 responded to our request and 4,753 agreed to participate in the survey. It is unknown whether those who did not respond were actually users of MRI, since they did not confirm their use. Of those who responded, about 80 percent were MRI users, 16 percent were not users, and 4 percent were not sure if they were users. The definition of MRI was included in the snowball sampling email, so the parameters of the imagery were clear. Examples of missions that collect MRI and products based on MRI were given to further illustrate the definition.

\section{User Survey}

We launched the survey in September of 2009 to all the people who had agreed to participate. We developed the survey in conjunction with experts at EROS to ensure that the technical details were accurate and that the instrument would gather information that would inform the USGS Land Remote Sensing (LRS) Program's distribution of imagery and future program requirements. One of the first decisions we made in this study was to conduct the survey online. An online survey is not appropriate for all populations because members cannot be assumed to have access to a computer, access to the Internet, an email account, or the technological skills necessary to complete a survey online. In this case, the population consisted of imagery users who must have access to a computer and the Internet to use the imagery, who have an email account for work purposes, and who must be at least somewhat technologically adept to use the imagery. Providing the survey online allowed an opportunity to ask only the questions relevant to each respondent through the use of automated logic patterns in the survey. Because the sample included both MRI users and non-users, we constructed a survey with questions tailored to four different types of users:

1) current Landsat users (who had used Landsat in the year previous to the survey),

2) current users of other types of MRI (who had used the imagery in the year previous to the survey),

3) past users of MRI (who had used the imagery at some point but not in the year previous to the survey), and

4) users of high- or low-resolution satellite imagery (who had used high- or low-resolution imagery at any time but who had never used MRI).

The set of questions for each of these users was considered a "survey path.” The answers to certain questions directed respondents to the appropriate survey paths, reducing the burden on 
respondents and collecting the most relevant information from each respondent. The last two groups were included because we were interested in understanding why users were using imagery other than MRI.

\section{Results}

\section{Analyses}

We analyzed the data in several different ways, including examining frequency data, chisquare analyses, t-tests, and contingent valuation analyses. Frequencies are reported for many results and chi-squares $\left(\chi^{2}\right)$ are reported where appropriate (for example, for comparisons between categorical variables). While many comparisons were made between various groups in the sample, differences between sectors (for example, academic institution, government, private business) were the most prevalent and significant; many of those differences are reported in this section. Chi squares are reported for each sector individually to illustrate differences between users in one sector versus users in all other sectors. T-tests are reported to compare means computed from scale variables. The contingent valuation analyses are described in detail in the Willingness to Pay for Landsat Imagery section.

\section{Statistical Significance and Interpretation}

Since the sample of MRI users in this study is not associated with a known population of MRI users, it is not possible to generalize the results for this sample to the population as a whole (as is typical when a random sample from a known population is drawn). For example, the majority of the sample was composed of users of Landsat imagery; however, that does not mean that the majority of all MRI users based in the U.S. use Landsat. However, the sample we obtained for this study is robust in terms of its size and diversity, such that comparisons among groups within the sample are appropriate. These comparisons are helpful to illustrate the diversity of users within the sample.

Because of the large sample size, the statistical power of this sample is very high, which may lead to results that are statistically significant but not meaningfully different (in other words, practically significant). Because of this, we report statistically significant results at the $\mathrm{p}<0.001$ level, rather than the more typical $\mathrm{p}<0.05$ level found in most behavioral and psychological research. Additionally, meaningful differences are estimated through measures of association, commonly called effect sizes. Effect size is a measurement of the amount of impact an independent variable has on a dependent variable (Murphy and Myors, 1998, p. 12). The effect sizes reported most frequently in this report are phi $(\Phi)$ and Cramer's V for chi-square analyses. Cohen (1988, p. 25-27, 79-80) provides the following guidelines for interpreting these effect sizes:

- $\quad$ a small effect $=0.1$ (for example, difference in mean height between 15- and 16-yearold girls),

- $\quad$ a medium effect $=0.3$ (for example , difference in mean height between 14- and 18year-old girls), and

- a large effect $=0.5$ (for example , difference in mean height between 13- and 18-yearold girls).

Following Cohen's recommendations on the interpretation of effect size for behavioral and psychological studies (1988, p. 25), we consider a statistically significant measure with an effect 
size of 0.1 or greater to indicate a meaningful difference for this study. All statistical results are located in the footnotes.

\section{Response Rate}

Over 2,500 people responded to the survey for a response rate of 53 percent $(n=2,523)$. This response rate is higher than average response rates for online surveys that have been found to be between 30-35 percent in several meta-analyses (for example, Lozar Manfreda and others, 2008; Sheehan, 2001; Shih and Fan, 2008). The high response rate may have been due to the initial contact during the snowball sampling, because pre-recruitment may have a positive impact on the response rates of online surveys (Lozar Manfreda and others, 2008). The high levels of interest and engagement respondents had regarding the topic may have contributed as well.

A non-response survey was conducted among those who did not respond to the initial survey to ascertain if those who did not respond were different than those who did. This short survey contained three questions: two related to user type (current Landsat users, current users of other MRI, etc.) and one to identify sector (government, private sector, academia, etc.). A total of 363 individuals responded to the non-response survey. These results were compared to those of the full respondent sample to determine differences. There were no practical differences between the distribution of user types among respondents and non-respondents. However, there were some differences in the distribution of the sectors among non-respondents and respondents. There were 14 percent fewer non-respondents in the academic sector, 10 percent more in the local government sector, and 6 percent more in the private sector. Because the sample was not random and the results are not being generalized to a population, the data were not weighted to account for these differences.

\section{Diversity of the Sample}

Determining the diversity of users within the sample is important in order to begin to understand what the larger user community might look like. These data cannot be generalized to the population at large, but the minimum amount of variety in the users and uses within the population can be gathered from these results. For example, users in the sample applied MRI in more than 35 application areas, but users in the population could be applying the imagery in even more application areas. In other words, while recognizing that this sample may not be representative of the population as a whole, we believe that the diversity of our sample provides a much needed baseline of types of users, uses, and valuations that can be expanded with further research.

One of the goals of the study was to reach users outside of the known community and the results indicate that goal was met. Almost half of the respondents currently using Landsat were not members of any type of remote sensing or GIS organization and, during the snowball sampling, almost 35 percent indicated they did not know any other users of MRI. Additionally, 45 percent of the current Landsat users did not obtain Landsat imagery from EROS in 2008 or 2009. These results indicate that many of the users in the sample were not part of the known groups within the population, such as professional organizations or users who procure imagery from EROS. Aside from reaching beyond the known user community, the sample was diverse in other ways. Users worked in seven different sectors and used the imagery in more than 35 different application areas. They conducted projects at scales from local to global in locations around the world. All of these data indicate that the sample was composed of a very diverse group of U.S.-based users. 


\section{User Types}

Current Landsat users (respondents who had used Landsat in the year prior to the survey) comprised more than half of the sample (fig. 1). Past MRI users comprised 15 percent of the respondents, users of high- or low-resolution imagery comprised 9 percent, and current other MRI users represented the smallest portion of the group with just under 7 percent. About 14 percent had never used any type of satellite imagery or did not know if they use MRI in their work. The latter two groups were not asked further questions in this survey because their lack of familiarity with the imagery would have made it difficult for them to answer the majority of the questions. About 24 percent of the respondents had used Landsat in the past; this group was made up of both current and past MRI users. The survey was structured to enable comparisons between current Landsat users and current users of other MRI. However, many other MRI users were unaware of the type of imagery (for example, Landsat, ASTER, SPOT) they used and other information from the survey (for instance, from open-ended questions) indicated that many of these “other" MRI users may actually have been using Landsat. Because of this and because the sample was predominantly made up of current Landsat users, the results in the remainder of the report refer to current Landsat users only, except where noted (see Appendix 2 for a summary of results for other user groups).

\section{Demographics}

The average current Landsat user in the sample was male, white, 47 years old, and highly educated. Three-quarters of the users were male, more than 90 percent were white, more than 80 percent were between 30 and 59 years old, and two-thirds had masters degrees or above. The predominant sector was academic institutions (33 percent), followed by private business (18 percent), and then federal (17 percent), state (16 percent), and local government (10 percent) (fig. 2). Only 4 percent of the users worked for non-profit organizations and less than 1 percent worked for tribes or nations. Anecdotally, we are aware of many tribes who are using the imagery and there were many tribal members identified in the initial web search, but very few responded to the survey. The small number prevented us from comparing respondents in the tribal sector with those in other sectors.

\section{Use of Landsat Imagery}

The first section of the survey established how the current Landsat users in the sample use the imagery, including types of imagery used, the scales and locations of projects, application areas, generally how the imagery is used, and the level of use in their work. Each question asked respondents to consider their use of Landsat in their work over the year previous to the survey.

\section{Types of Imagery}

About 40 percent of respondents indicated they had used only Landsat imagery in the past year. The remaining 60 percent of Landsat users indicated they used a mix of imagery, with 


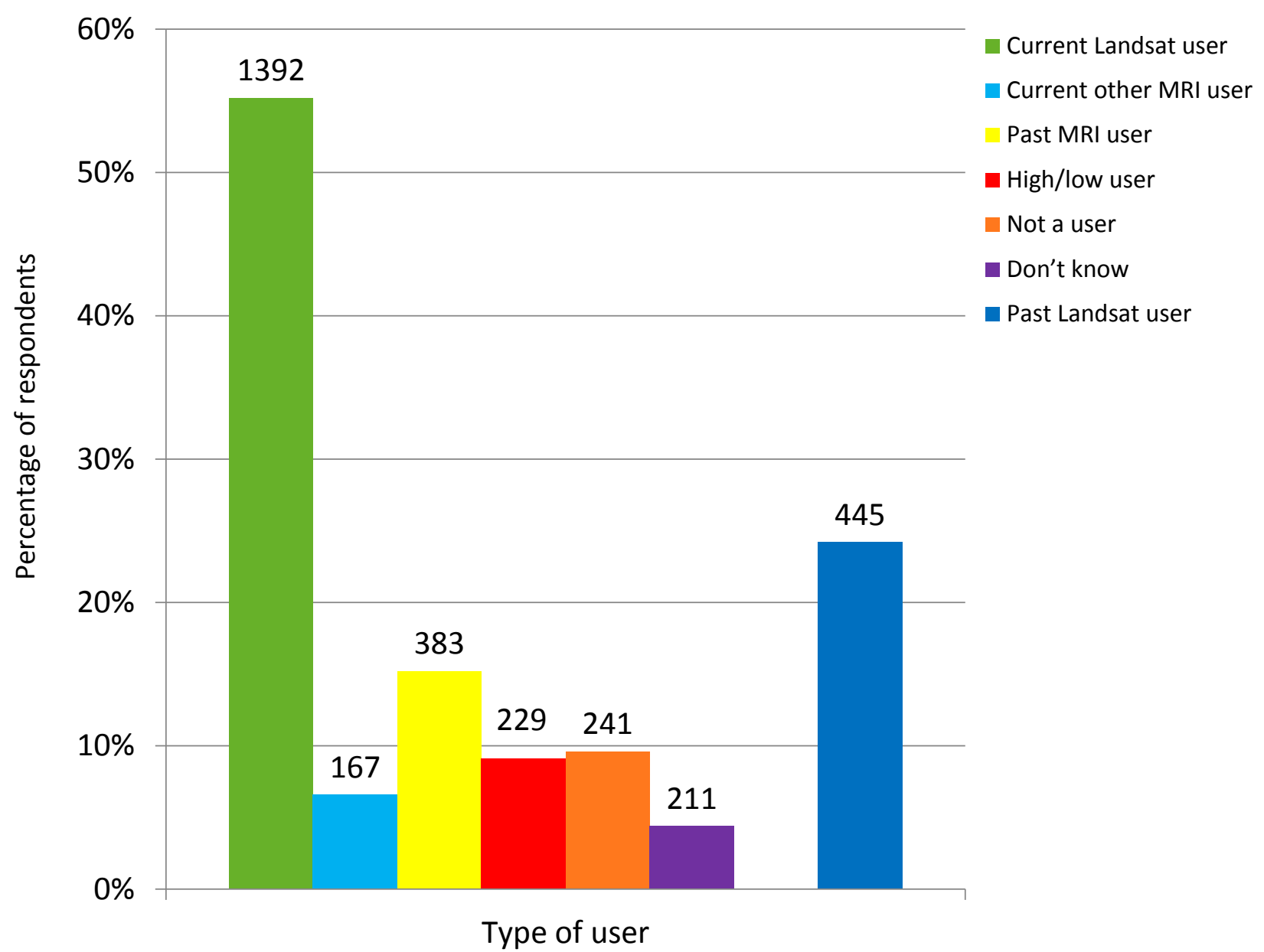

Figure 1. Respondents by type of satellite imagery used.

about half coming from Landsat, followed by 11 percent from Terra (ASTER), 8 percent from SPOT (HRVIR, HRG, HRS), and 3 percent from Resourcesat (LISS, AWiFS) (table 1). One percent or less came from ALOS (AVNIR-2) or CBERS (CCD) on average. ${ }^{4}$ About 6 percent of the imagery came from other satellites and about 16 percent of the imagery was from unknown satellite sources.

There are interesting differences among sectors in the imagery used for those using a mix of MRI (table 1). Respondents in the local government sector used the least amount of Landsat imagery on average (31 percent) and used the most imagery from unknown satellite sources (43 percent). Academic users using a mix of MRI, on the other hand, obtained 65 percent of their imagery from Landsat satellites and only 5 percent from unknown satellite sources.

\section{Scales and Locations of Projects}

Respondents' projects that relied on Landsat ranged from local to global scales in locations around the world. Two-thirds of respondents (66 percent) worked only on projects

\footnotetext{
${ }^{4}$ ASTER - Advanced Spaceborne Thermal Emission and Reflection Radiometer, SPOT - Satellite Pour l'Observation de la Terre, HRVIR - High-Resolution Visible and Infrared, HRG - High-Resolution Geometrical, HRS - High-Resolution Stereo, LISS - Linear Imaging Self Scanner, AWiFS - Advanced Wide Field Sensor, ALOS - Advanced Land Observing Satellite, AVNIR-2 - Advanced Visible and Near Infrared RadiometerType 2, CBERS - China-Brazil Earth Resources Satellite, CCD - Charge Coupled Device
} 


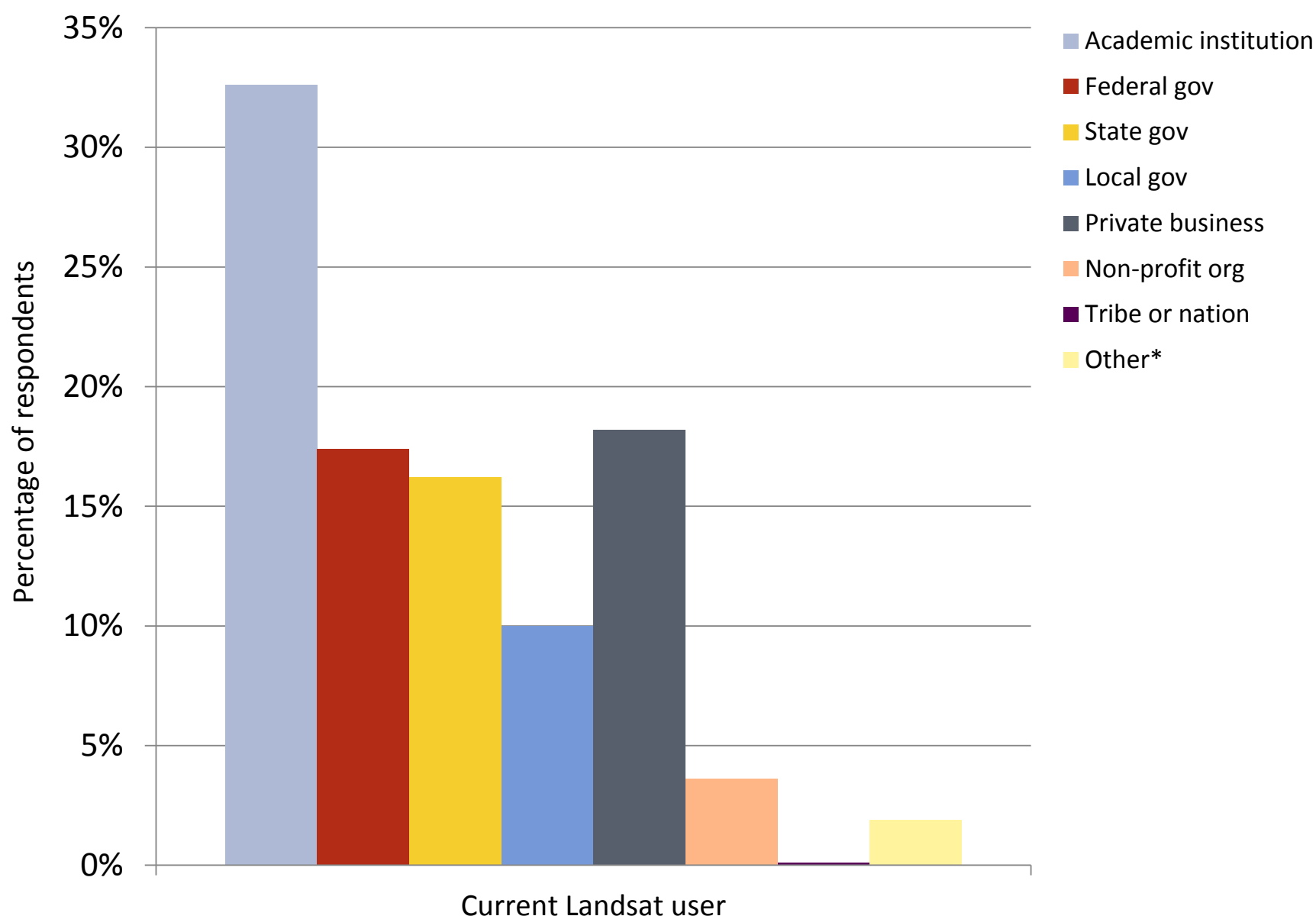

*Other sector is composed of respondents who wrote in more than one sector.

Figure 2. Sectors of current Landsat users.

Table 1. Mean percentage of imagery used in the year previous to the survey among current Landsat users who used a mix of moderate-resolution imagery.

\begin{tabular}{lccccccc}
\hline Imagery & $\begin{array}{c}\text { All } \\
\text { sectors }\end{array}$ & $\begin{array}{c}\text { Academic } \\
\text { institution }\end{array}$ & $\begin{array}{c}\text { Federal } \\
\text { government }\end{array}$ & $\begin{array}{c}\text { State } \\
\text { government }\end{array}$ & $\begin{array}{c}\text { Local } \\
\text { government }\end{array}$ & Private & $\begin{array}{c}\text { Non-profit } \\
\text { organization }\end{array}$ \\
\hline Landsat & $54 \%$ & $65 \%$ & $57 \%$ & $49 \%$ & $31 \%$ & $48 \%$ & $57 \%$ \\
Terra & $11 \%$ & $15 \%$ & $12 \%$ & $7 \%$ & $5 \%$ & $10 \%$ & $11 \%$ \\
SPOT & $8 \%$ & $7 \%$ & $8 \%$ & $9 \%$ & $8 \%$ & $7 \%$ & $9 \%$ \\
Resourcesat & $3 \%$ & $2 \%$ & $7 \%$ & $2 \%$ & $2 \%$ & $3 \%$ & $4 \%$ \\
ALOS & $1 \%$ & $<1 \%$ & $1 \%$ & $1 \%$ & $<1 \%$ & $2 \%$ & $2 \%$ \\
CBERS & $<1 \%$ & $<1 \%$ & $1 \%$ & $<1 \%$ & $<1 \%$ & $1 \%$ & $1 \%$ \\
Other & $6 \%$ & $5 \%$ & $6 \%$ & $5 \%$ & $10 \%$ & $8 \%$ & $3 \%$ \\
Unknown & $16 \%$ & $5 \%$ & $8 \%$ & $26 \%$ & $43 \%$ & $21 \%$ & $13 \%$ \\
\hline Total & $100 \%$ & $100 \%$ & $100 \%$ & $100 \%$ & $100 \%$ & $100 \%$ & $100 \%$ \\
\hline
\end{tabular}


located in the U.S., while 28 percent have worked on projects in both the U.S. and internationally (fig. 3). Far fewer users (6 percent) worked only on projects located internationally. Respondents in the state ${ }^{5}$ and local ${ }^{6}$ government sectors were more likely to have worked only in the U.S. than users in other sectors, while academic respondents were more likely to have worked in both the U.S. and internationally. ${ }^{7}$

Respondents predominantly worked at the regional (for example, multi-state or province) scale or lower (fig. 4). Respondents in local government were more likely to have worked at a local scale than other users ${ }^{8}$ and those in state government were more likely to have worked at the state scale. ${ }^{9}$

\section{Application Areas}

A list of 37 application areas was developed by examining previous surveys of satellite imagery users, as well as through consultations with experts at EROS. Respondents were asked to select their primary application of Landsat from the list, which included an "other" category

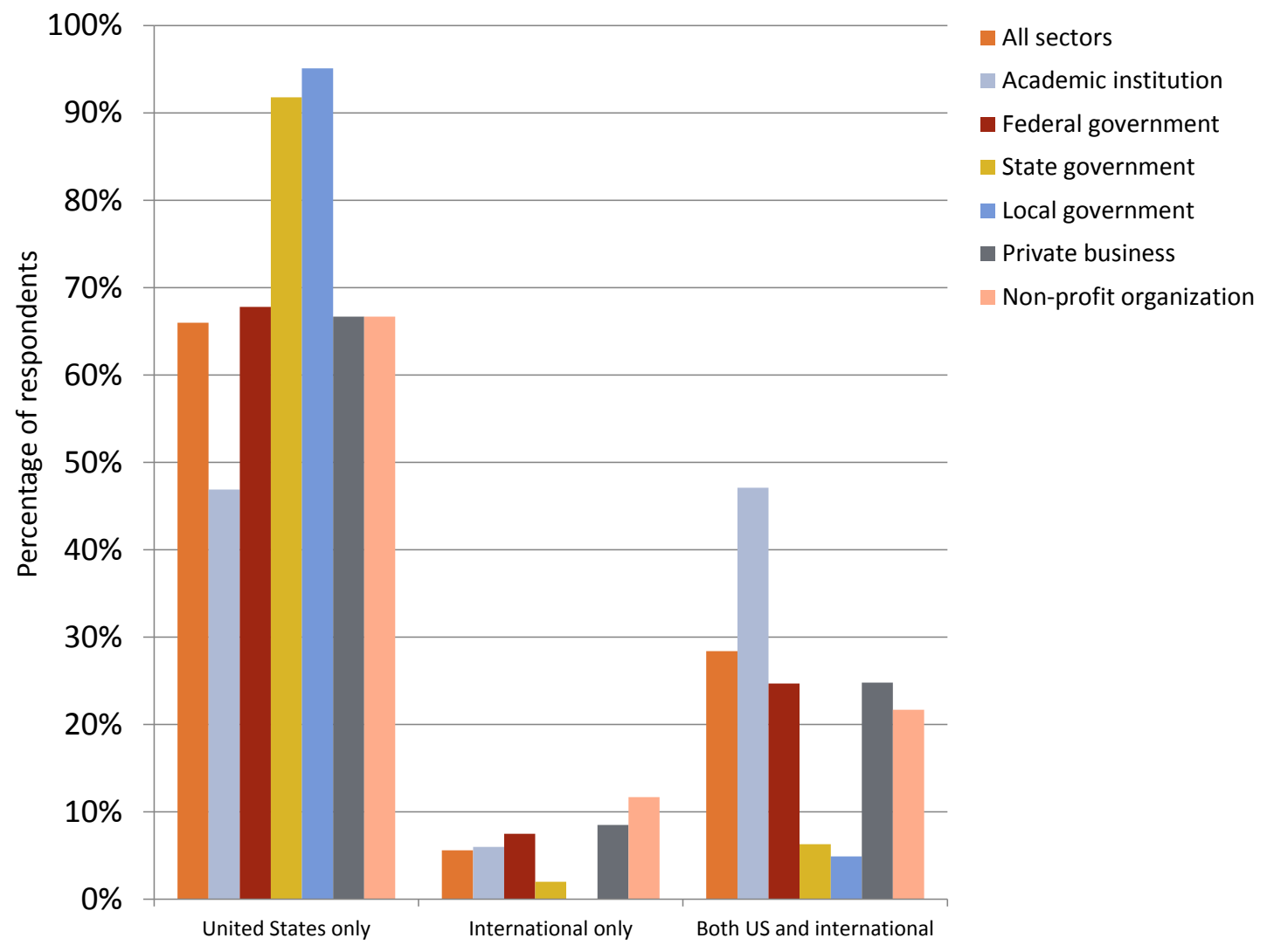

Figure 3. Locations of projects using Landsat imagery in year previous to survey.

${ }^{5} \chi^{2}=74.98$, Cramer's $\mathrm{V}=0.233$

${ }_{7}^{6} \chi^{2}=55.11$, Cramer's V $=0.200$

${ }^{7} \chi^{2}=127.88$, Cramer's $V=0.304$

${ }_{0}^{8} \chi^{2}=30.18, \Phi=0.148$

${ }^{9} \chi^{2}=42.26, \Phi=0.175$ 


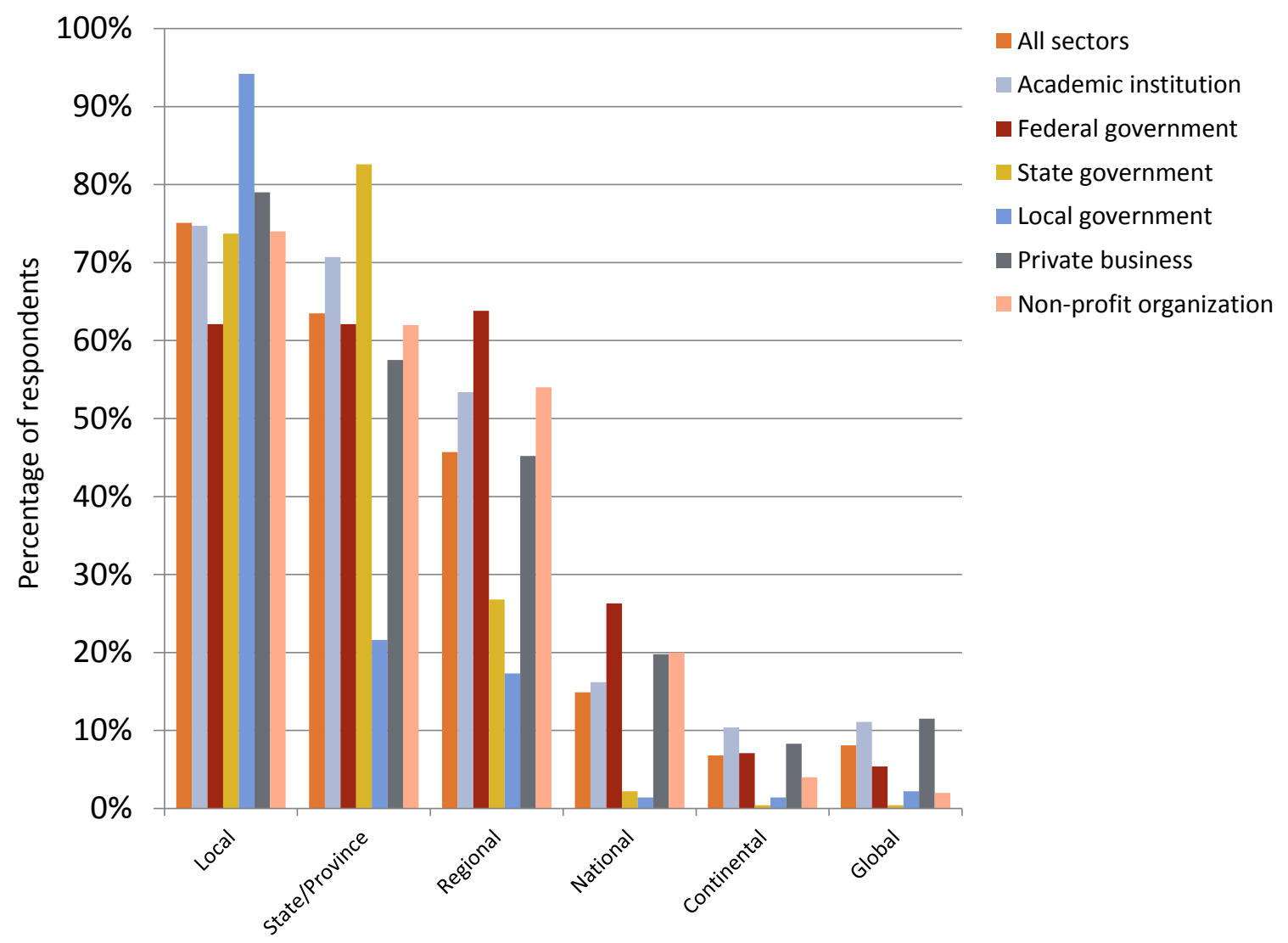

Figure 4. Scales of projects using Landsat imagery in year previous to survey.

where they could write in an application area (table 2). They were then asked to select as many secondary applications as they wished from the same list. The 37 applications were collapsed into nine larger categories for the purposes of analysis (table 2). Environmental science and management applications were the most commonly selected with more than 40 percent of respondents choosing one (fig. 5). Land use/land cover (17 percent) was the second most common application, followed by planning and development (11 percent), education (8 percent), and agriculture ( 8 percent). Land use/land cover is different than the rest of the applications since users can be working in environmental science, planning and development, or any number of other application areas where land use/land cover analyses could be conducted. Of those who chose land use/land cover as their primary application, the most common secondary applications were environmental sciences, followed by planning and development applications, such as urbanization and rural and urban planning and development.

There were clear differences among sectors in these primary applications (fig. 5). Respondents in the academic sector were more likely to apply Landsat imagery in the area of education ${ }^{10}$, whereas those in the Federal government were more likely to have applied Landsat in agriculture ${ }^{11}$ and environmental sciences. ${ }^{12}$ Those in local government were more likely to

\footnotetext{
${ }^{10} \chi^{2}=162.70, \Phi=0.343$

${ }^{11} \chi^{2}=28.70, \Phi=0.144$

${ }^{12} \chi^{2}=19.98, \Phi=0.120$
} 
Table 2. Applications of Landsat imagery among current Landsat users.

\begin{tabular}{|c|c|}
\hline Collapsed applications for analysis & Individual applications \\
\hline \multirow[t]{2}{*}{ Agriculture } & Agricultural forecasting \\
\hline & Agricultural management/production/conservation \\
\hline \multirow[t]{11}{*}{ Environmental sciences and management } & Biodiversity conservation \\
\hline & Climate science/change \\
\hline & Coastal science/monitoring/management \\
\hline & Ecological/ecosystem science/management \\
\hline & Fish and wildlife science/management \\
\hline & Fire science/management \\
\hline & Forest science/management \\
\hline & Geology/glaciology \\
\hline & Range/grassland science/management \\
\hline & Recreation science/management \\
\hline & $\begin{array}{l}\text { Water resources (for example, watershed management, water } \\
\text { rights, hydrology) }\end{array}$ \\
\hline Land use/land cover & Land use/land cover \\
\hline \multirow[t]{5}{*}{ Planning and development } & Assessments and taxation \\
\hline & Engineering/construction/surveying \\
\hline & Rural planning and development \\
\hline & Urban planning and development \\
\hline & Urbanization \\
\hline \multirow[t]{6}{*}{ Commercial } & $\begin{array}{l}\text { Cultural resource management (for example, archaeology, } \\
\text { anthropology) }\end{array}$ \\
\hline & Real estate/property management \\
\hline & Software development \\
\hline & Telecommunications \\
\hline & Transportation \\
\hline & Utilities \\
\hline \multirow[t]{3}{*}{ Education } & Education: K-12 \\
\hline & Education: university/college \\
\hline & Technical training (for example, workshops, short courses) \\
\hline \multirow[t]{4}{*}{ Human needs } & Emergency/disaster management \\
\hline & Hazard insurance (for example, crop, flood, fire) \\
\hline & Humanitarian aid \\
\hline & Public health \\
\hline \multirow[t]{3}{*}{ Legal/security } & Defense/national security \\
\hline & Environmental regulation \\
\hline & Law enforcement \\
\hline Oil/gas/minerals & Oil and gas/mineral exploration/extraction \\
\hline
\end{tabular}




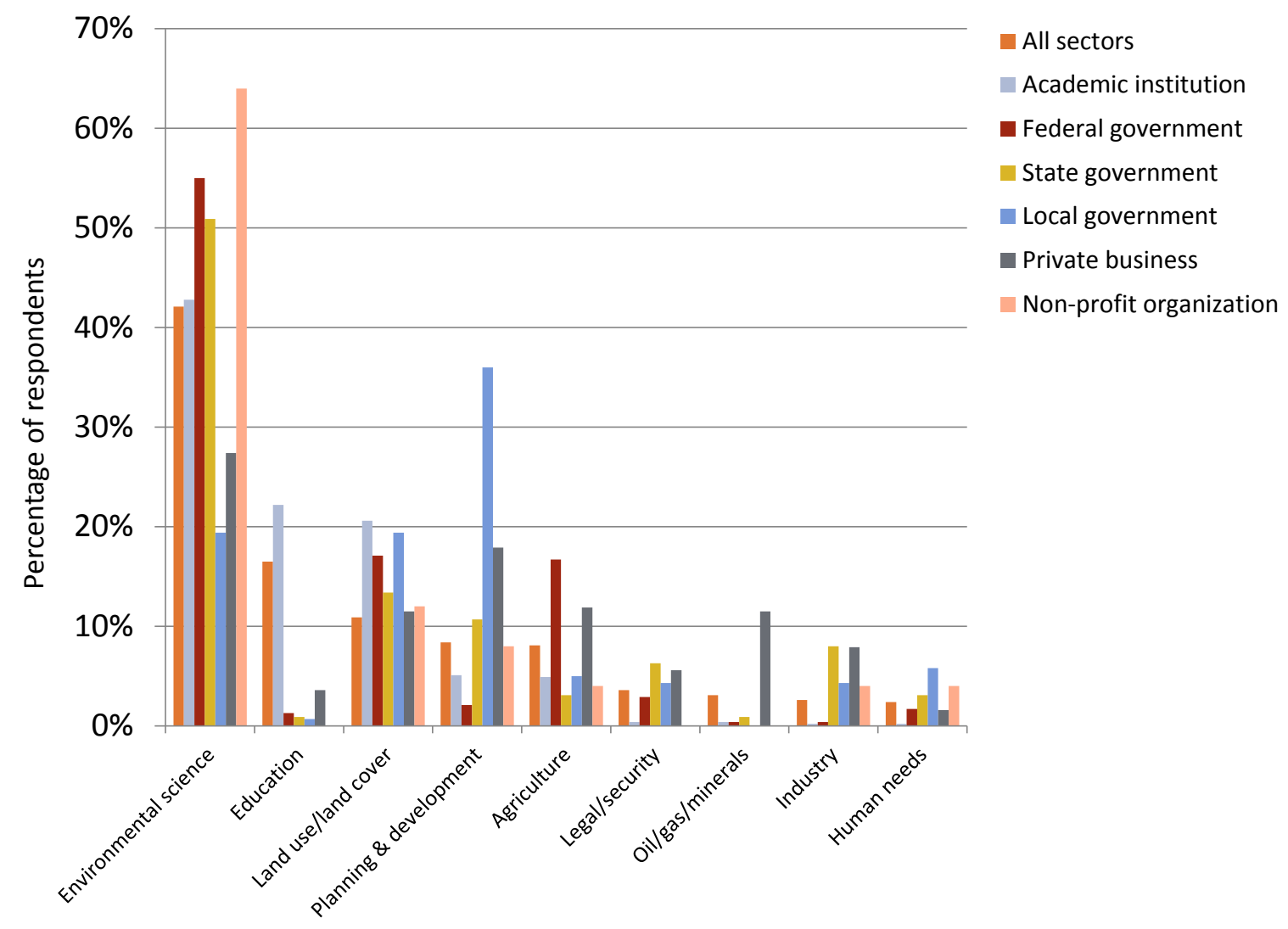

Figure 5. Applications of Landsat imagery in projects conducted in year previous to survey.

have used Landsat for planning and development. ${ }^{13}$ Those in the private sector were also more likely to apply Landsat in planning and development ${ }^{14}$, as well as in commercial applications ${ }^{15}$ and oil/gas/minerals exploration and extraction. ${ }^{16}$

In addition to these current application areas, there may be new and unique uses in the future. We asked respondents to write in uses they foresee in the next five years. Common responses included change detection using time series analyses, integration with other imagery or products, and climate change monitoring and awareness. For example, one respondent believed that new uses will "mostly come from the power of comparing the long catalog with new observations, especially associated with urbanization and global warming induced changes." There were also several comments that emphasized the potential for new uses, rather than specifying any. For instance, one respondent stated, "The more that these (now free) data get into the hands of previously unengaged users, the greater the potential for discovery of new uses.”

\footnotetext{
${ }^{13} \chi^{2}=98.69, \Phi=0.267$

${ }_{14} \chi^{2}=14.89, \Phi=0.104$

${ }_{15} \chi^{2}=17.43, \Phi=0.112$

${ }^{16} \chi^{2}=96.47, \Phi=0.264$
} 


\section{How Imagery is Used}

To get a sense of how respondents are using MRI in general, we asked them to describe their overall work with the imagery (fig. 6). The majority of respondents (91 percent) used the imagery to answer questions and (or) solve problems, processed imagery for themselves or others (62 percent), and made decisions based on the imagery (57 percent). Only 19 percent developed algorithms, 12 percent provided or sold imagery or value-added products, and 2 percent developed commercial software. By sector, those in academia were more likely to process imagery ${ }^{17}$ and develop algorithms ${ }^{18}$, whereas those in local ${ }^{19}$ and state ${ }^{20}$ government were less likely to use the imagery in these ways. Those respondents in the private sector were more likely to provide or sell imagery ${ }^{21}$ and develop commercial software. ${ }^{22}$

\section{Level of Landsat Use in Work}

While all the current Landsat users in the sample used Landsat, whether exclusively or in conjunction with other imagery, the percentage of their work that relied on Landsat over the past year varied. In order to effectively describe level of use, we categorized respondents as heavy, medium, or light users. Light users relied on Landsat for 30 percent or less of their work, medium users relied on it for 31-70 percent of their work, and heavy users relied on it for 71 percent or more of their work. Overall, almost two-thirds (63 percent) of respondents were classified as light users, 18 percent as medium users and 15 percent as heavy users (fig. 7). There were some differences between these use levels among sectors. Respondents in the academic sector were more likely to be heavy users ${ }^{23}$ than respondents in other sectors, while those in local $^{24}$ and state ${ }^{25}$ government were more likely to be light users. For example, only 1 percent of respondents in local government were classified as heavy users, compared to 10-21 percent of users in other sectors. In contrast, 81 percent of local government users were classified as light users, which is 7-30 percent higher than in any other sector.

While this categorization is helpful in understanding level of use, it does not indicate dependence on Landsat. A light user could rely on Landsat for a critical operational use that accounts for less than 30 percent of their work, but which would be compromised if Landsat imagery was not available. We did not explicitly ask about dependence in this survey, though there are some proxies for dependence that will be discussed further in the Value of Landsat Imagery section.

\section{"Local Users"}

After examining the results outlined above, it became clear there was a group of users who were different from the others. We found a group of users characterized by sector, project scale, and project location, dubbed "local users," who represented about 25 percent of the current Landsat users in the sample. They worked for local or state governments applying imagery in

\footnotetext{
${ }^{17} \chi^{2}=17.78, \Phi=0.113$

${ }_{18} \chi^{2}=62.33, \Phi=0.212$

${ }_{19}$ Process imagery $-\chi^{2}=22.13, \Phi=-0.127$; Develop algorithms $-\chi^{2}=19.50, \Phi=-0.119$

${ }^{20}$ Process imagery $-\chi^{2}=17.00, \Phi=-0.111$; Develop algorithms $-\chi^{2}=32.19, \Phi=-0.153$

${ }^{21} \chi^{2}=47.80, \Phi=0.186$

${ }^{22} \chi^{2}=40.47, \Phi=0.171$

${ }^{23} \chi^{2}=51.67$, Cramer's V $=0.193$

${ }^{24} \chi^{2}=32.22$, Cramer's V $=0.153$

${ }_{25} \chi^{2}=16.77$, Cramer's $V=0.110$
} 


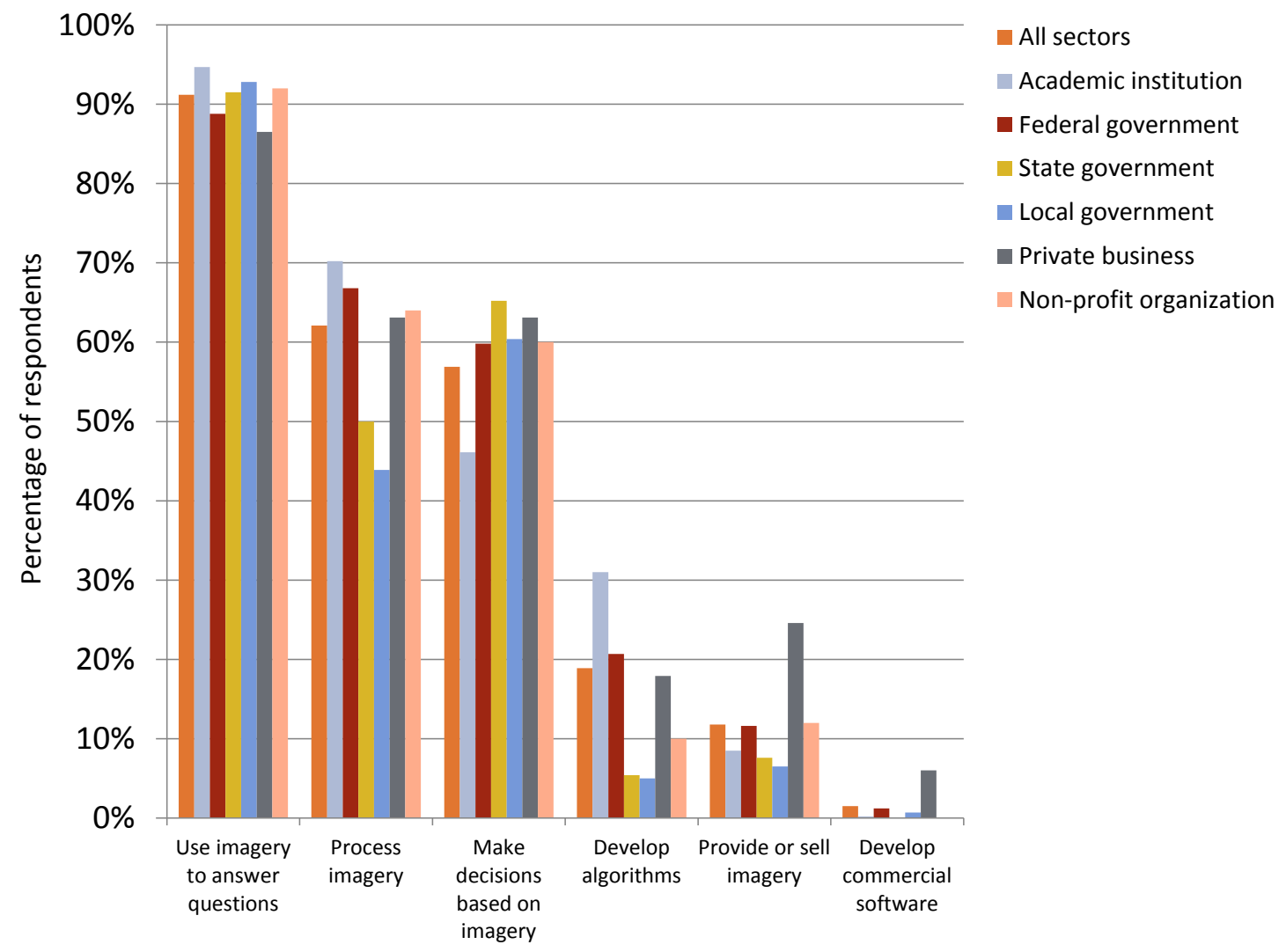

Figure 6. General use of moderate-resolution imagery among current Landsat users.

local-scale projects located in the U.S. There are several indications that this group may be a less technical user group than other users in the sample. They were less likely to process imagery or develop algorithms, more likely to be a light Landsat user, and less likely to know the satellite source of all the imagery they use. They also appeared to be less involved in the professional user community, as they were less likely to be a member of a remote sensing or GIS-related organization.

Discussion: Use of Landsat Imagery

Overall, Landsat imagery was the primary MRI used by these respondents, but the uses of the imagery varied greatly among these respondents. In the year previous to the survey, respondents worked on projects at all different scales in locations around the world. Each of the 37 application areas on the list was selected by a minimum of two respondents as their primary application. The general uses of MRI indicate that there was a mix of technical abilities among the respondents in the sample, with some respondents processing the imagery or developing algorithms or software, and others using it in less technical ways to answer questions or make decisions. There were also respondents using Landsat at all levels in their work, with some using Landsat in all their work and some using it in very little of their work. Taken together, these results reveal a diverse sample of users whose responses provide a baseline for exploring the uses in the population as a whole. 


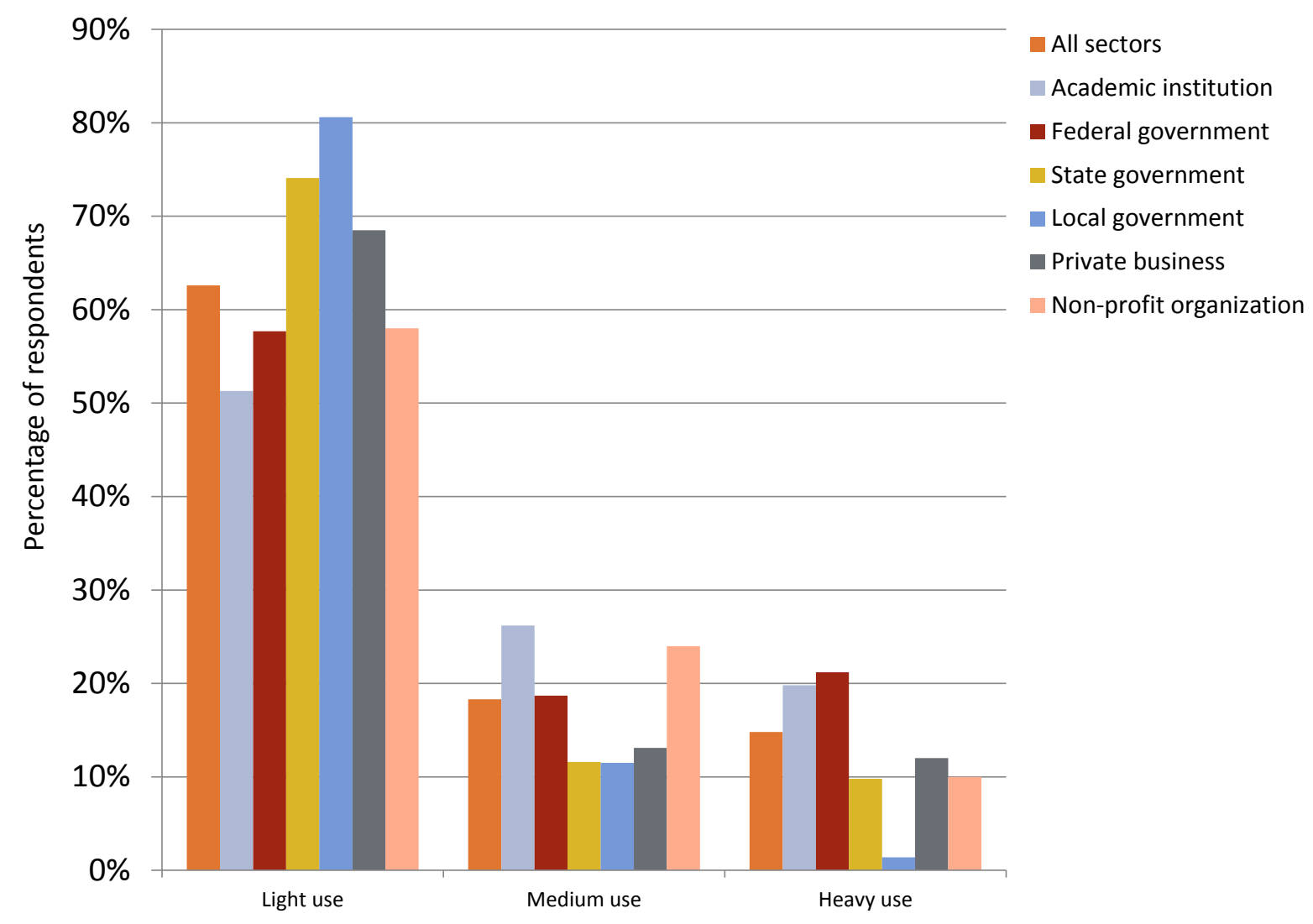

Figure 7. Level of use of Landsat imagery among current Landsat users(as measured by the percentage of respondents' work that used Landsat imagery in year previous to survey).

Comparing the results by sector demonstrated that there are significant differences among the sectors in this sample when it comes to the uses of Landsat imagery. For instance, the existence of the group of "local users" demonstrates that many respondents in state and local governments were dissimilar from respondents in other sectors. The results indicate that the different roles and goals of each sector guide the uses of Landsat imagery by respondents in those sectors.

\section{Changes in Use of Landsat Imagery over Time}

Many events over the recent history of the Landsat mission may have impacted people's use of the imagery. To track how these events may impact use, respondents were asked how their use of Landsat changed over the past 10 years and how they envisioned it would change over the next 5 years.

About 80 percent of respondents said their use increased or stayed the same in the past 10 years and will increase or stay the same in the next 5 years (fig. 8). Of the users who stated their use increased or would increase, the majority (66-80 percent) chose both changes in work and cost as reasons. Respondents cited many other reasons as well, some under the control of data providers (for example, availability, accessibility), but most outside of the control of data providers (for example, fixed attributes of the sensor like spatial resolution, new 


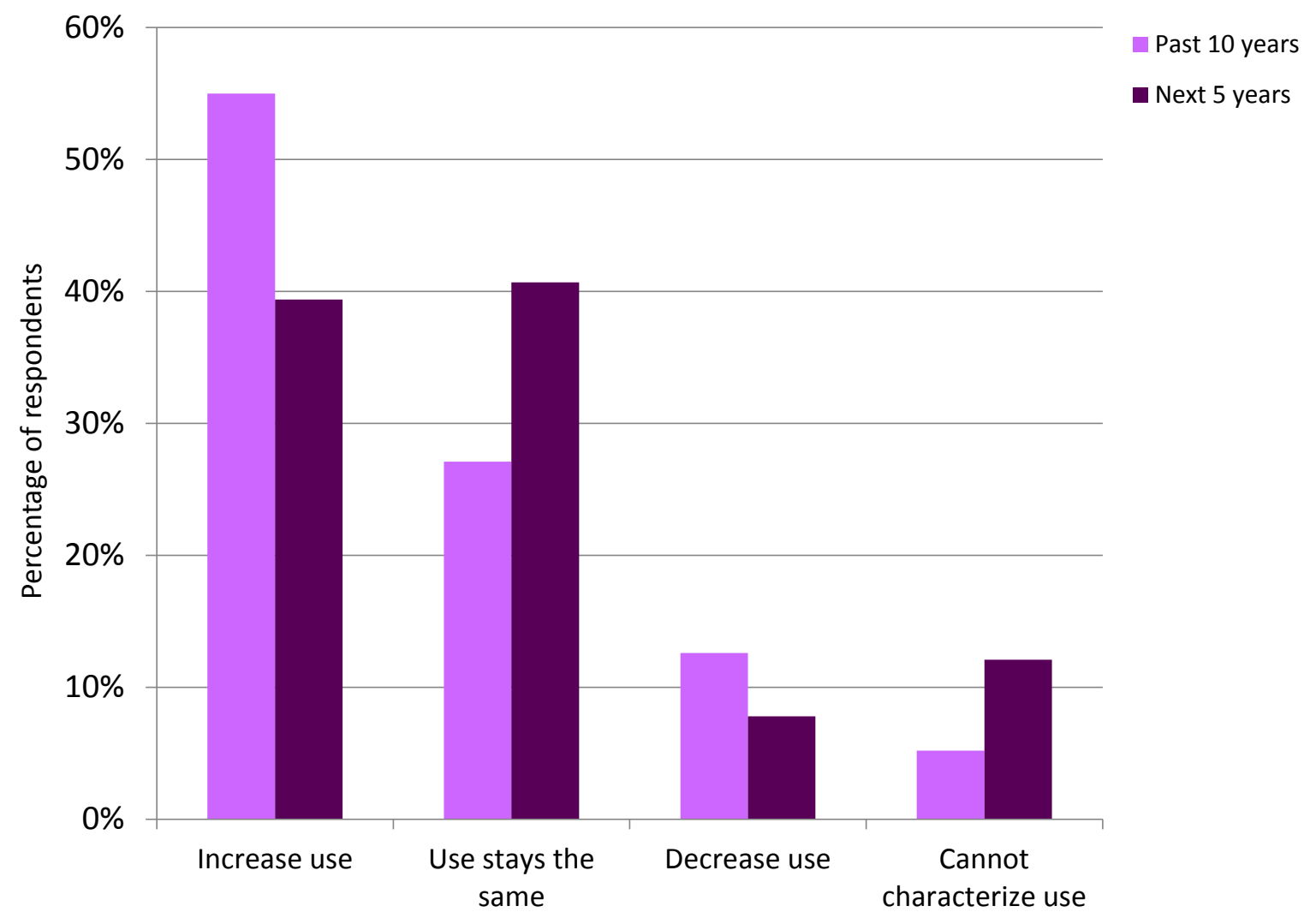

Figure 8. Actual and predicted changes in use of Landsat imagery in past 10 years and next 5 years among current Landsat users.

applications/uses, more demand for imagery from clients, improvements in hardware used to process imagery, more training or more knowledgeable staff). Some respondents based their future use entirely on the successful launch of LDCM and the provision of new usable imagery. By sector, respondents in academia were more likely to cite more affordable imagery as a reason for increasing use in both the past ${ }^{26}$ and the future, ${ }^{27}$ whereas those in local government were less likely to cite more affordable imagery in both the past ${ }^{28}$ and the future. ${ }^{29}$

Given the recent USGS implementation of the no-cost data policy for Landsat imagery and the anticipated launch of LDCM, we hypothesized that respondents who ceased using Landsat in the past may begin using it again in the future. Two-thirds of those who had used Landsat in the past said they foresee using it in the future (fig. 9). However, when asked what would make them more likely to use MRI in the future, the most common reasons given were changes in work (71 percent of respondents), improved spatial resolution (52 percent), and lower

\footnotetext{
${ }^{26} \chi^{2}=40.44, \Phi=0.216$

${ }^{27} \chi^{2}=24.06, \Phi=0.198$

${ }^{28} \chi^{2}=12.35, \Phi=-0.119$

${ }^{29} \chi^{2}=13.08, \Phi=-0.146$
} 


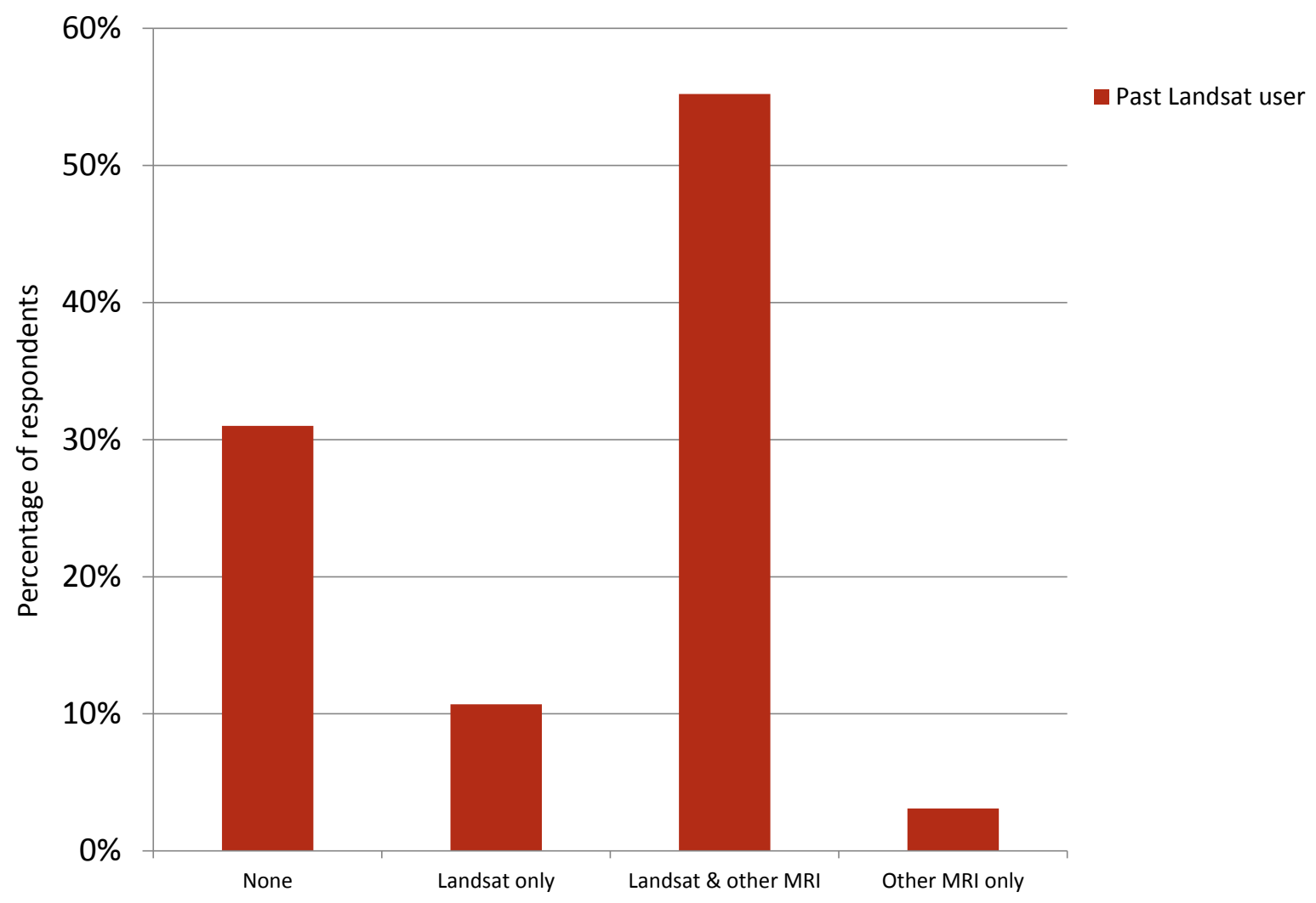

Figure 9. Predicted future use of Landsat and other moderate-resolution imagery among past Landsat users.

cost (33 percent). This indicates that cost may not be the driving factor if past users decide to use Landsat imagery again.

Less than 13 percent of respondents said their use of Landsat imagery had decreased or would decrease (fig. 8). Of the respondents who said their use decreased over the past 10 years, most cited spatial resolution, the attractiveness of other imagery, and changes in work as reasons. Those who indicated their future use will decrease also cited the attractiveness of other imagery and spatial resolution, but they were furthermore concerned with data quality and temporal resolution. Almost all respondents (96 percent) who said they will decrease use in the future cited reasons outside of the control of USGS, including other imagery being more attractive, attributes of the sensor, changes in work and new Landsat data not being available in the case of a data gap. Less than 20 percent cited reasons within the control of USGS, including availability, accessibility, and licensing/distribution restrictions. Interestingly, SLC-off on Landsat 7 appears to have had a minimal impact on respondents who said they had decreased use in the past 10 years-only 69 users cited it as a reason. In response to SLC-off, about two-thirds of these respondents replaced Landsat 7 imagery with Landsat 5 imagery (fig. 10). More than half replaced Landsat 7 imagery with other MRI, just under a third used gap-filled or SLC-off Landsat 7, and less than a fifth used some other kind of data as a replacement. 


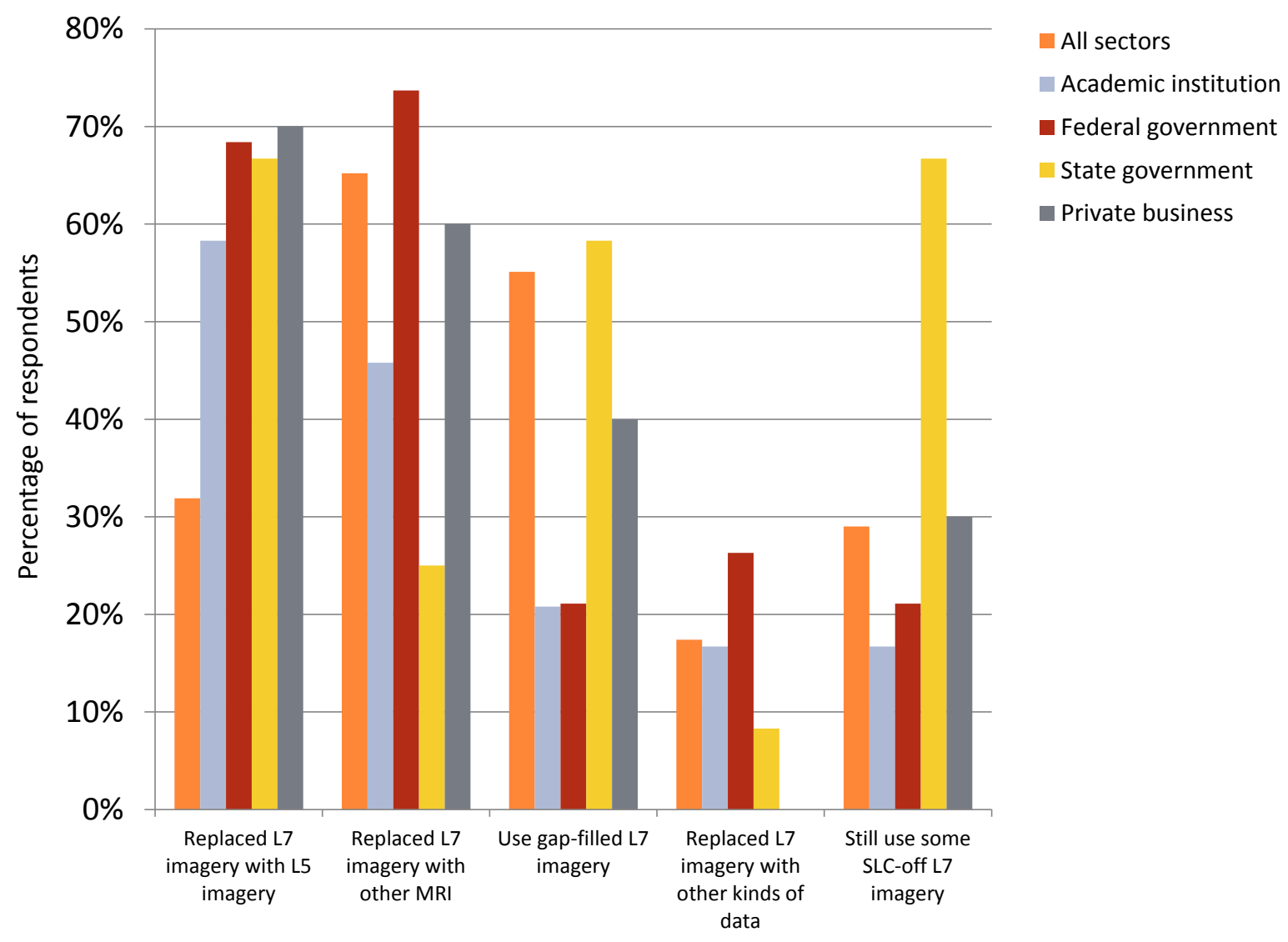

Figure 10. Reactions to Landsat 7 imagery with SLC-off among current Landsat users who indicated their Landsat use had decreased over the past 10 years.

\section{Discussion: Change in Use of Landsat Over Time}

Regardless of whether respondents' use increased or decreased in the past or will change in the future, the majority of reasons given are outside of the control of USGS, except for more affordable imagery, which was cited only for increasing use. This indicates that most of the current Landsat users in this sample are satisfied with the provision of imagery by USGS, even if they may want to see changes to the imagery itself or to the sensors that capture the images. More affordable imagery was cited by the majority of respondents as a reason for increasing use, except among respondents in the local government sector. Given that local governments are often faced with restricted budgets, this is a surprising result. However, it is possible that users in this sector have not traditionally paid for any of the data they use, thus making cost a non-issue. There is also the possibility that they are not aware that Landsat is available at no cost now (there were several comments in the survey to that effect) and so do not know that Landsat has become more affordable. Among respondents who decreased use in the past 10 years, the SLC-off on Landsat 7 was not a major factor. Given that the majority of these users replaced Landsat 7 imagery with Landsat 5 imagery, this indicates that the loss of Landsat 5 before the launch of LDCM may impact some users more than others. 


\section{Impacts of No Cost Data Policy}

The entire archive of Landsat imagery became available at no cost at the beginning of 2009. To determine the impacts of this policy change, we asked respondents about their imagery acquisitions before and after the policy change (calendar year 2008 and calendar year 2009, respectively). Respondents did not have to personally download the data, but did have to use it in their projects. First we asked where respondents had acquired imagery in 2008 and 2009 and provided a list of possible sources. USGS EROS was the most common source of the data in both years with more than 45 percent of respondents obtaining data there, followed by the Internet in general (more than 20 percent of users) (fig. 11). A quarter of the respondents did not know where their imagery came from in both years, indicating that perhaps they were not personally acquiring the imagery. We were particularly interested in whether respondents who did not acquire imagery from EROS in 2008 had done so in 2009 in response to the change in policy. However, less than 10 percent of the respondents behaved in that manner and an almost equal amount acquired imagery from EROS in 2008 but not in 2009 (fig. 12).

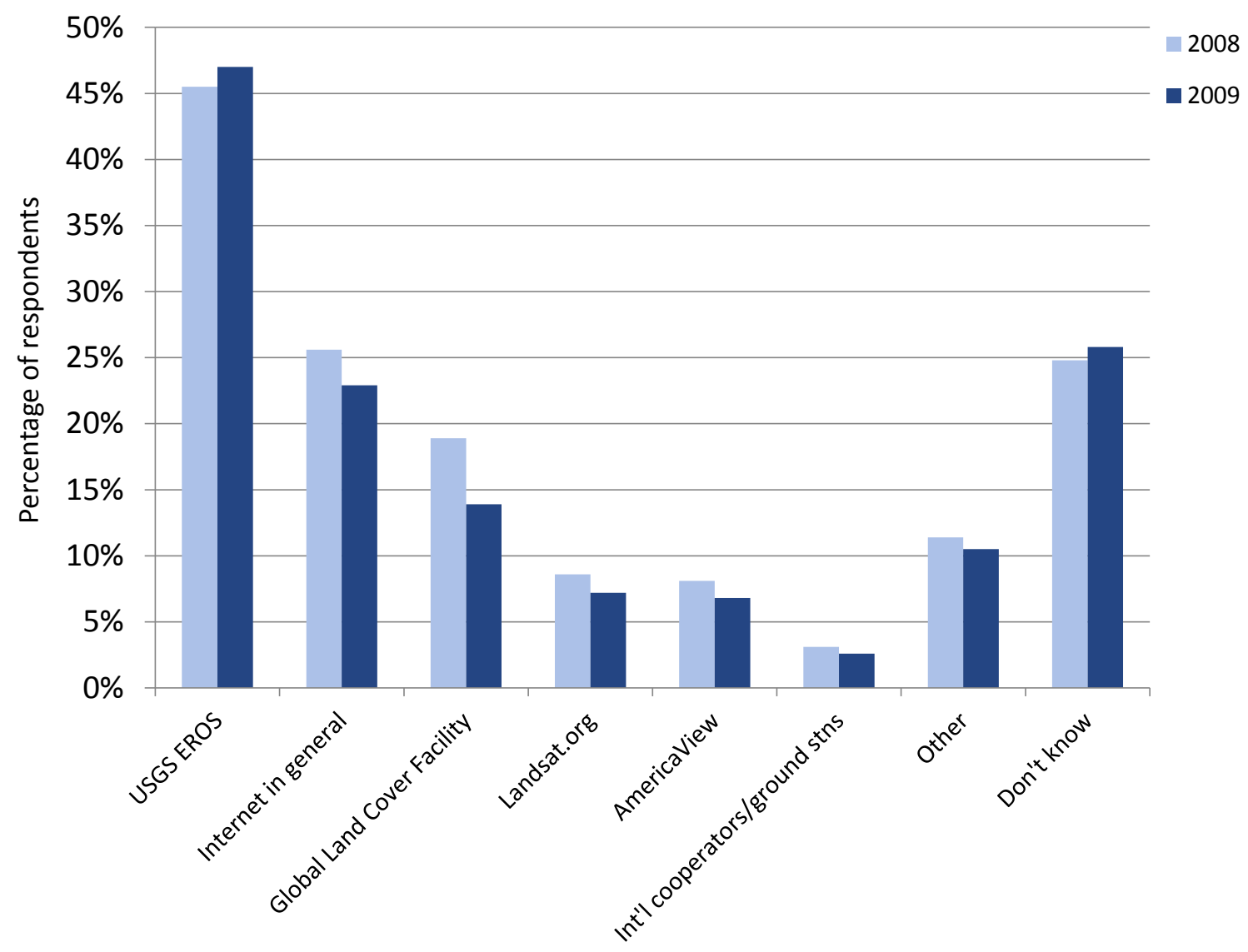

Figure 11. Sources used by current Landsat users to obtain Landsat imagery before and after the imagery became available at no cost from USGS EROS (2008 versus 2009). 


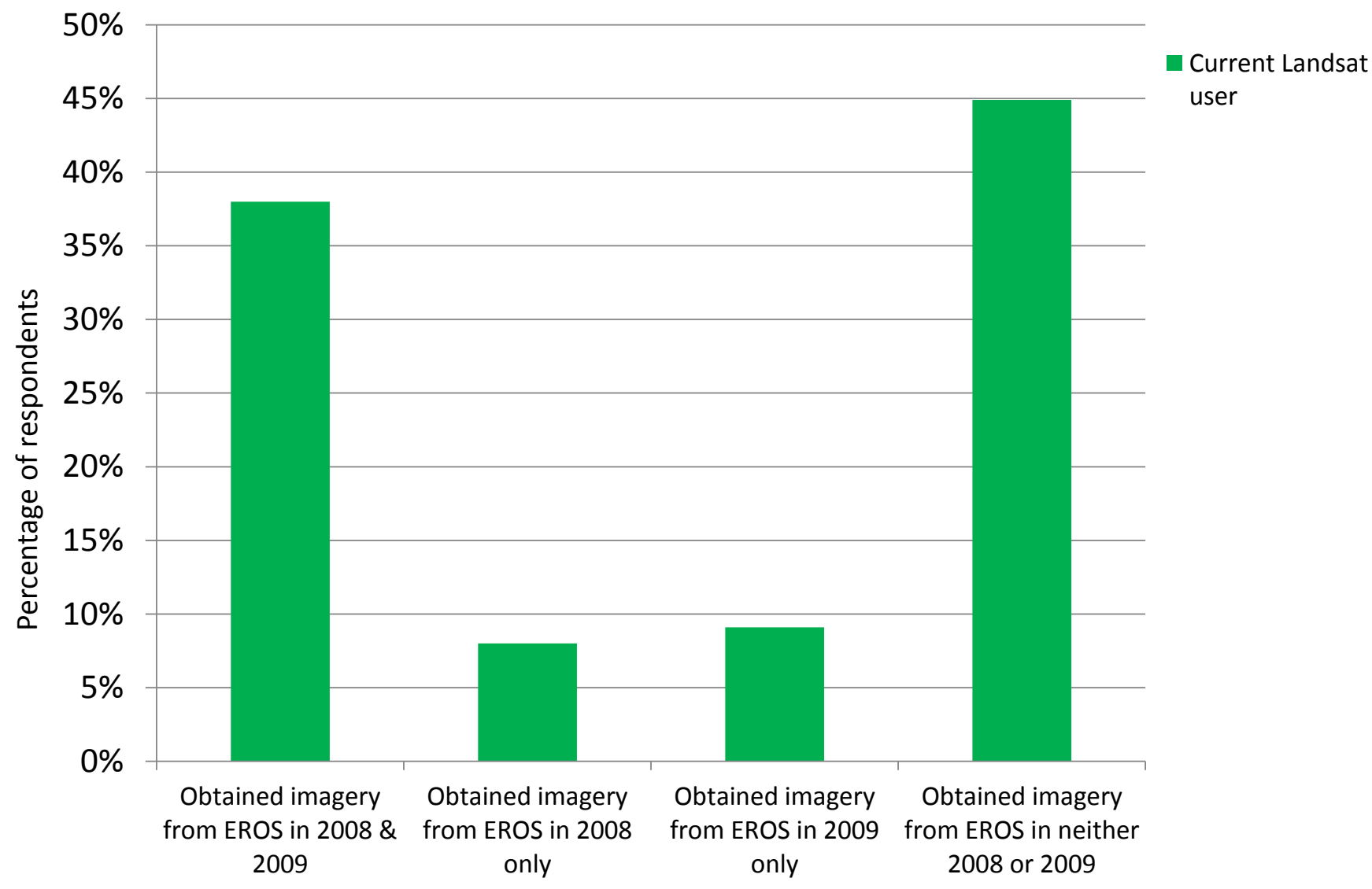

Figure 12. Current Landsat users' patterns of acquisitions of Landsat imagery from USGS EROS before and after it became available at no cost (2008 versus 2009).

Though there were few differences in where respondents acquired imagery in 2008 and 2009, there were significant changes in the number of scenes acquired, the percentage of those scenes acquired from EROS, and the dollar amount spent on scenes between the two years (table 3). A paired samples t-test was conducted on those three variables for which data from both 2008 and 2009 were available for any given respondent. Between 900 and 1,000 respondents provided information for both years for each of the three variables. Statistically significant results were found for each variable. In 2009, the average number of scenes acquired increased by 45 percent and the percentage of those scenes acquired from EROS increased by 6 percent. The average amount spent on Landsat imagery in 2009 was one-fifth of what was spent in 2008.

\section{Discussion: Impacts of No Cost Data Policy}

Even though there were few changes overall in where respondents acquired their Landsat imagery before and after the no cost policy went into effect, there were significant changes in how many scenes were acquired and how much was spent on those scenes in 2008 versus 2009. Interestingly, the effect sizes (Cohen's $d$ for within-subjects analyses in this case) were very 
Table 3. Acquisitions of Landsat imagery before and after it became available at no cost (2008 versus 2009) by current Landsat users.

\begin{tabular}{lcccc}
\hline Acquisition variable & $\mathbf{2 0 0 8}$ mean & $\mathbf{2 0 0 9}$ mean & $\boldsymbol{t}$ & $\boldsymbol{p}$ \\
\hline Number of scenes acquired & 95 & 138 & -3.95 & $<0.001$ \\
Percentage of scenes acquired from EROS & $41 \%$ & $47 \%$ & -4.93 & $<0.001$ \\
Dollars spent on imagery & $\$ 5,117$ & $\$ 1,163$ & 5.28 & $<0.001$ \\
\hline
\end{tabular}

small for each comparison (Cohen's $\mathrm{d}<0.2$ ). ${ }^{30}$ This means that, for instance, the number of scenes acquired in 2008 accounts for very little of the variation in the number of scenes acquired in 2009. In this case, the lack of connection between the variables over the two years indicates that other factors, such as the availability of the imagery at no cost or changes in work, had a substantial impact on the acquisitions of the respondents in 2009.

\section{Value of Landsat Imagery}

In economic terms, the value of information is equal to what individuals would pay for that information (Macauley, 2006). The value depends on the uncertainty of the situation in which the information will be used, the importance of the outcome of the situation, the cost of using the information, and the cost of an appropriate substitute. Macauley $(2005,2006)$ notes that there are several ways the economic value of information has been examined, including output or productivity measures, hedonic price studies, contingent valuation studies, and measurement of societal benefits. However, societal benefits can be difficult to measure economically, especially when the realized value is in relation to a nebulous, but important, concept like quality of life. Additionally, the comprehensive value of Landsat may always be elusive, given the widespread use of the imagery in applications like Google Earth and the difficulty in finding all direct and indirect users of the imagery. All of these factors emphasize the importance of measuring the value of information provided by Landsat imagery in multiple ways.

We used four approaches to estimate the value of Landsat to this sample of Landsat users. First, we explored the importance of Landsat imagery to respondents, as well as their satisfaction with the imagery. Second, we asked about the environmental and societal benefits, including impacts on decisionmaking, from projects that used Landsat. Third, we asked what respondents would do if Landsat imagery was no longer available and how it would impact their work. Lastly, we utilized a method called contingent valuation to determine respondents' willingness to pay for imagery equivalent to Landsat in the event that there is a gap in imagery provision.

\section{Importance and Satisfaction}

Exploring the importance of Landsat imagery to users is one way to approach value. More than 80 percent of the respondents said the imagery is somewhat or very important to their work (fig. 13). Once again, there are differences by sector. Respondents in academia were more

\footnotetext{
${ }^{30}$ Cohen (1988, p. 25-26) provides the following guidelines for interpreting Cohen's d: a small effect $=0.2$, a medium effect $=0.5$, and a large effect $=0.8$.
} 


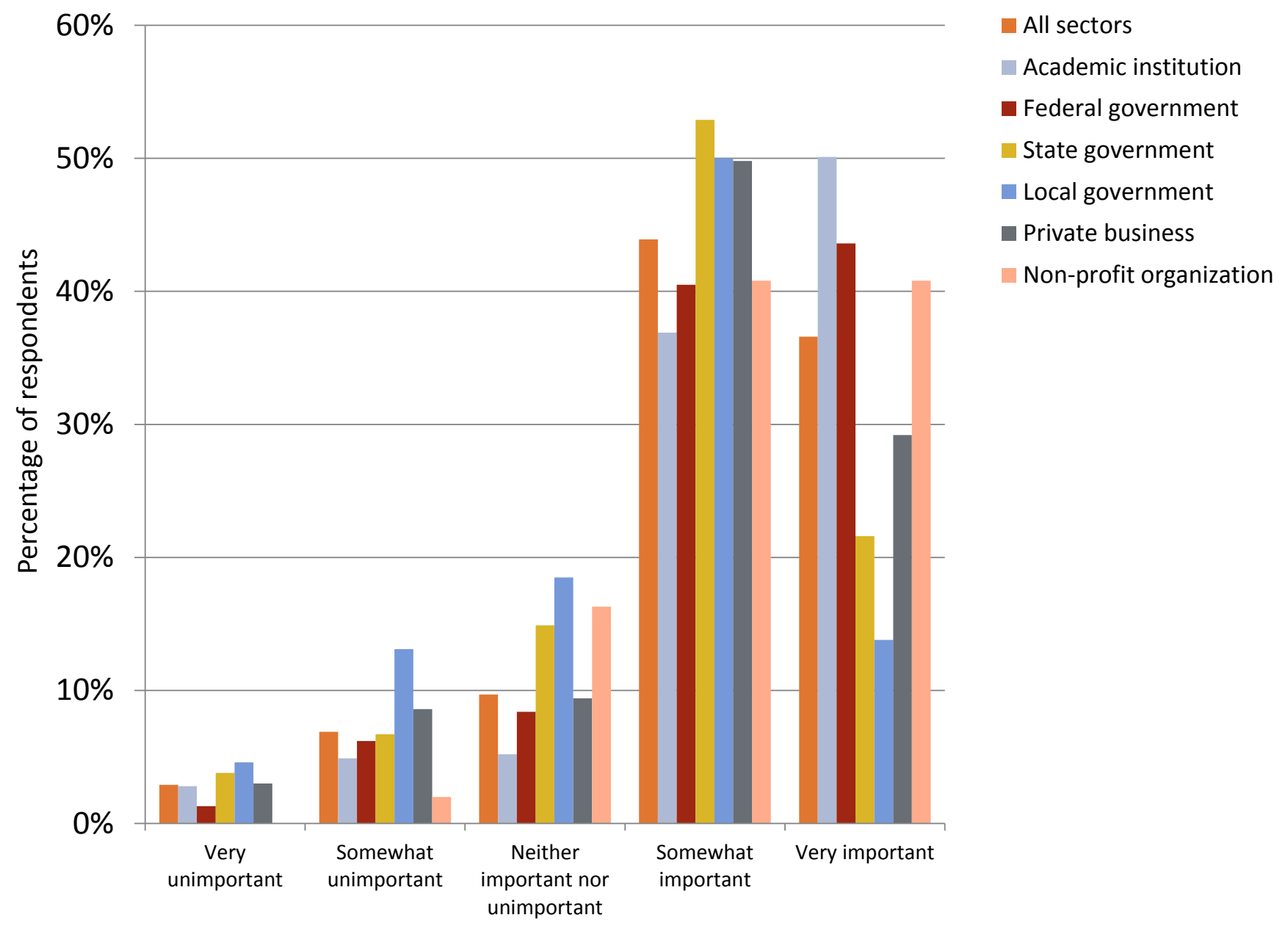

Figure 13. Importance of Landsat imagery to the work of current Landsat users.

likely to think the imagery is very important to their work ${ }^{31}$ while those in the state ${ }^{32}$ and local ${ }^{33}$ government sectors were less likely to say Landsat is very important.

We also asked respondents to rate how important certain attributes of MRI are in determining which type of MRI to use and how satisfied they are with those same attributes as they occur in Landsat imagery. This is a common approach in marketing research to assess how well a product is meeting the needs of customers (Martilla and James, 1977). From this data, we created an importance-performance framework that maps satisfaction on the $\mathrm{X}$ axis by importance on the Y-axis (fig. 14). It allows us to look at where things are going well and where room for improvement exists. All of the Landsat attributes we asked about fall in the "keep up the good work" quadrant; in other words, on average, users think all of the attributes measured are important and they are satisfied with the provision of those attributes. The highest ratings were for availability, accessibility, and cost, which indicate that users are satisfied with how the imagery is being provided. There were no significant differences in these ratings by sector,

\footnotetext{
${ }^{31} \chi^{2}=55.71$, Cramer's V $=0.207$

${ }^{32} \chi^{2}=27.06$, Cramer's V $=0.144$

${ }^{33} \chi^{2}=42.35$, Cramer's V $=0.181$
} 


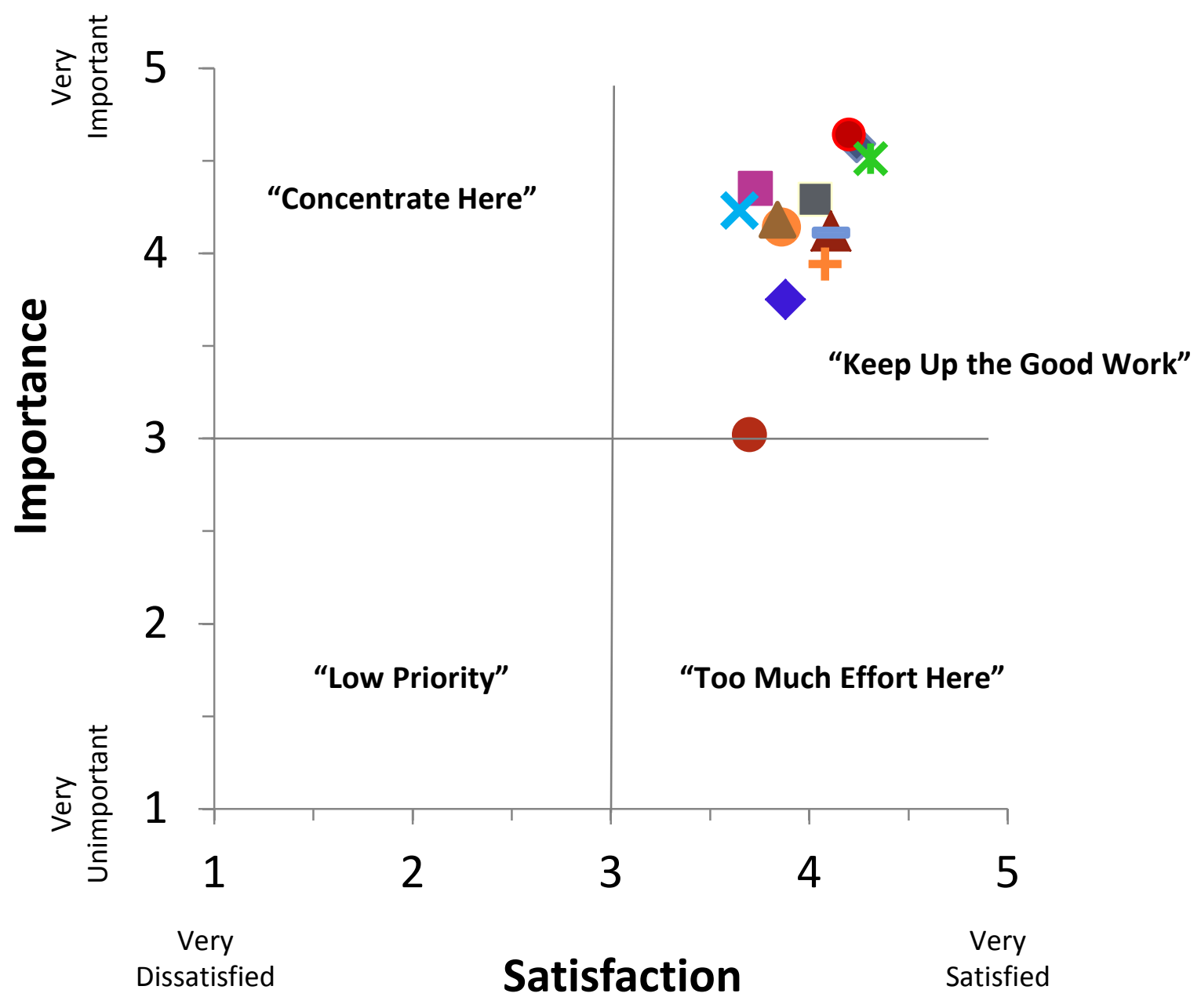

Accessibility

$\triangle$ Area/footprint of individual scene

- Cost

- Delivery time

- Global coverage

- Spatial resolution

* Temporal resolution/frequency of coverage
Archive/continuity

Availability

Data quality assessments

- Ease of use

- Licensing/distribution restrictions

$\triangle$ Spectral resolution

Figure 14. Mean importance of and mean satisfaction with certain attributes of Landsat imagery among current Landsat users. 
application area, or any other variables, except for global coverage. Global coverage is different from the rest of the attributes because it was rated as having only average or neutral importance. We believe this is driven by the large portion of the sample that is doing work only in the U.S. without a need for international coverage. This is supported by the fact that global coverage is more important for people working internationally: only 23 percent of people working in just the U.S. think it is important, whereas 63 percent of people working internationally think it is important. ${ }^{34}$

\section{Benefits of Landsat}

The benefits of Landsat are not well documented in a systematic way. In an effort to gather more information about the benefits of Landsat, we asked a series of open-ended questions where respondents could write in their responses. Open-ended questions were chosen because no comprehensive list of benefits has been developed and we wanted to give respondents the opportunity to provide their own ideas about benefits. The responses were examined for repeating themes and then categorized into those themes. When asked how projects using Landsat had affected decisionmaking, many respondents mentioned impacts on policy. One respondent noted, "We have been able to come up with evidence to change small town policy and challenge politics. Good science is hard to beat." Another mentioned, "One of my papers was presented before members of Congress and used to influence legislation." Others noted that the use of Landsat made the results of their project easier to understand for decisionmakers. One respondent said, "I can display the results of my work against the imagery background, which makes it easier for people to understand." Another stated, "Landsat has helped others visualize spatial relations much more clearly."

A second question inquired about the environmental and (or) societal benefits that have come about as a result of projects that used Landsat. Protecting or conserving the environment, including improving environmental conditions, reducing impacts on the environment and assessing environmental impacts and change over time, was the most common benefit noted by respondents. One respondent noted that the “...preservation of trees and greenspace, especially forested areas, has measurably improved water and air quality, reduced stormwater runoff, and improved quality of life within the communities I have worked with.” Another stated that "perhaps Landsat['s] greatest utility is the long timeframe that can be accessed." Increasing human safety was also a common theme among the responses. One respondent stated that "the benefit to society I would think is priceless. I say this because if map details come together to help save a life in an emergency (and they have, many times), how much would that life be worth if it wasn't saved?” Another respondent said that projects using Landsat imagery had resulted in a "safer transportation system" and the "ability to quickly assess detour routes in case of emergency." A third benefit frequently mentioned was improved decisionmaking, whether it was more effective, more informed, or more inclusive. One respondent noted, "The greatest benefit I have perceived is that providing stakeholders with spatial data gives them a sense of empowerment to make decisions. The unexpected benefit is that map data act to build consensus among groups with competing interests. Seeing actual data tends to dissolve apparent symbolic differences and helps different groups work together on issues of environmental management.”

\footnotetext{
${ }^{34} \chi^{2}=276.58$, Cramer's V $=0.427$
} 
If Landsat Imagery Was No Longer Available...

Another way to examine the value of a good is to explore the impacts that would occur if it ceased to exist. We asked users what would happen to their work if both new and archived Landsat imagery was no longer available. We assumed users could:

- discontinue some or all of their work;

- continue their work without substituting other imagery or information; and (or)

- use other imagery or information as a substitute in their work.

About half of the users would discontinue at least some of their work (table 4). Half would continue at least some of their work without substituting other imagery or information. Just over 75 percent of the users would substitute either other imagery, other data sets, or field work for at least some of their work.

Of those who would discontinue some of their work $(n=693), 45$ percent would discontinue a small percentage of their work (30 percent or less), 32 percent would discontinue a medium percentage (31-70 percent), and 23 percent would discontinue a high percentage (71 percent or more). In fact, 11 percent would discontinue more than 90 percent of their work if Landsat was no longer available, which indicates a strong dependence on the imagery. There were some differences by sector on this variable. Academic users were more likely to discontinue a medium to high percentage of their work than users in other sectors ${ }^{35}$.

Table 4. Percentages of current Landsat users who would take each of three actions if Landsat imagery was no longer available.

\begin{tabular}{lccc}
\hline Action taken if Landsat was no longer available & $\begin{array}{c}\text { Would take } \\
\text { action }\end{array}$ & $\begin{array}{c}\text { Would not take } \\
\text { action }\end{array}$ & $\begin{array}{c}\text { Don't } \\
\text { know }\end{array}$ \\
\hline Substitute other imagery or information in work & $76 \%$ & $4 \%$ & $20 \%$ \\
$\begin{array}{l}\text { Discontinue work } \\
\begin{array}{l}\text { Continue work without substituting other imagery or } \\
\text { information }\end{array}\end{array}$ & $51 \%$ & $28 \%$ & $21 \%$ \\
\hline
\end{tabular}

Of those who would substitute ( $n=1,067)$, almost 90 percent would use other imagery, about two-thirds would use other data sets, and slightly fewer would use fieldwork (table 5). Given that fieldwork tends to be more expensive and time consuming than using imagery or other data, this seems to indicate that fieldwork might be the only viable substitute to provide certain types of data. This may be because other similar appropriate imagery does not exist or is not affordable or attainable. If users indicated they would use substitute imagery, they were asked what imagery they would prefer regardless of budget constraints. Then they were asked what imagery they would most likely acquire given their current budget constraints. More than half would choose the same imagery regardless of budget constraints - the most common choice was Terra (ASTER), followed by SPOT (HRVIR, HRG, HRS), and then Resourcesat (LISS, AWiFS) (fig. 15). However, 40 percent would choose different imagery based on whether they were constrained by budget. For those respondents, SPOT (HRVIR, HRG, HRS) was the preferred imagery without budget constraints, but Terra (ASTER) was the imagery that the majority would be most likely to acquire with budget constraints (fig. 16).

${ }^{35} \chi^{2}=49.98$, Cramer's V $=0.216$ 
Table 5. Percentages of current Landsat users who would use each of three types of information as substitutes for Landsat imagery if it was no longer available.

\begin{tabular}{lccc}
\hline Type of substitute information & Would use & Would not use & Don't know \\
\hline Other imagery & $89 \%$ & $1 \%$ & $10 \%$ \\
Other data sets & $69 \%$ & $15 \%$ & $16 \%$ \\
Fieldwork & $63 \%$ & $25 \%$ & $12 \%$ \\
\hline
\end{tabular}

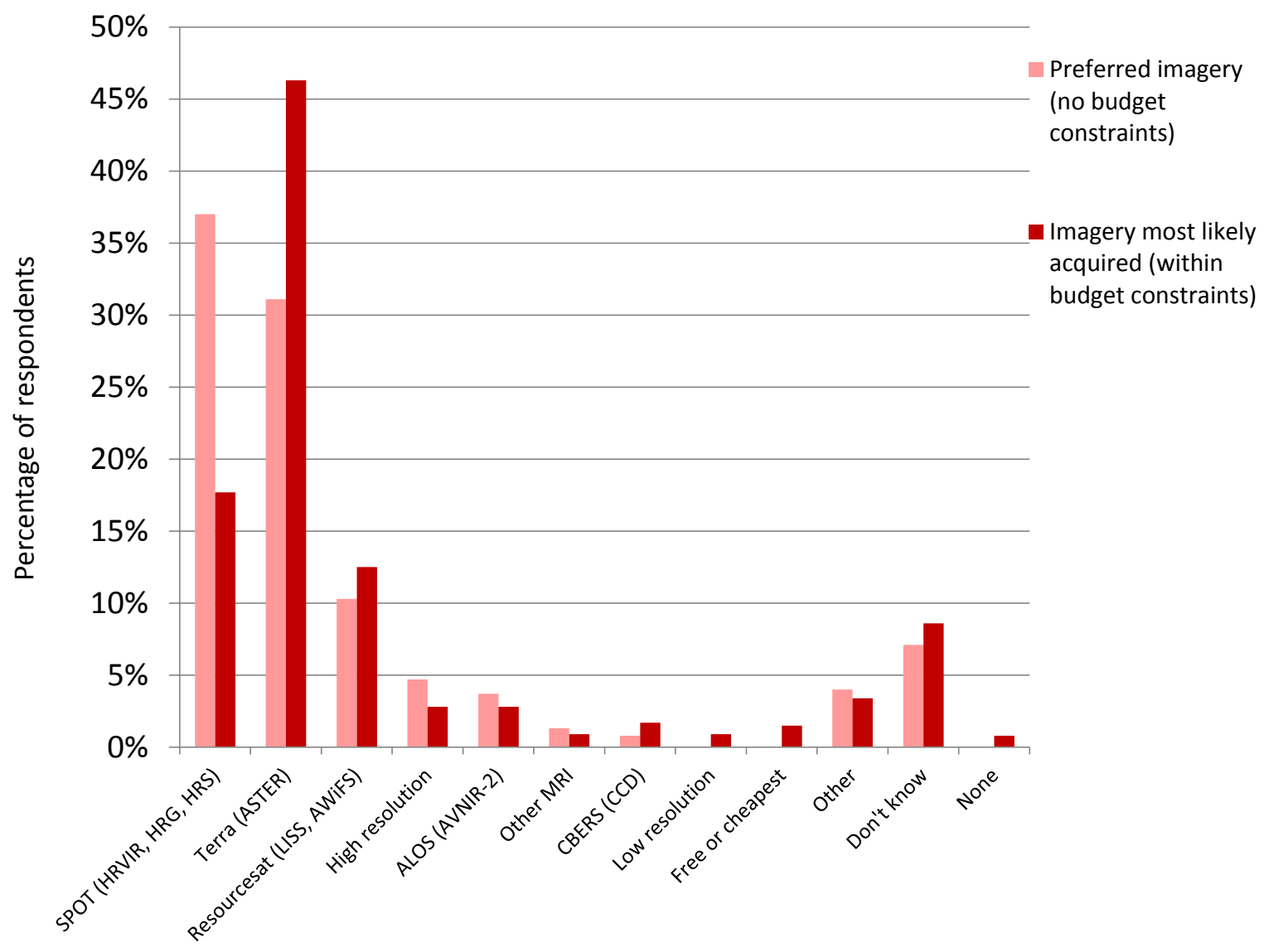

Figure 15. Preferred imagery versus imagery most likely to be acquired within budget constraints among current Landsat users who would substitute for Landsat imagery if it was no longer available. 


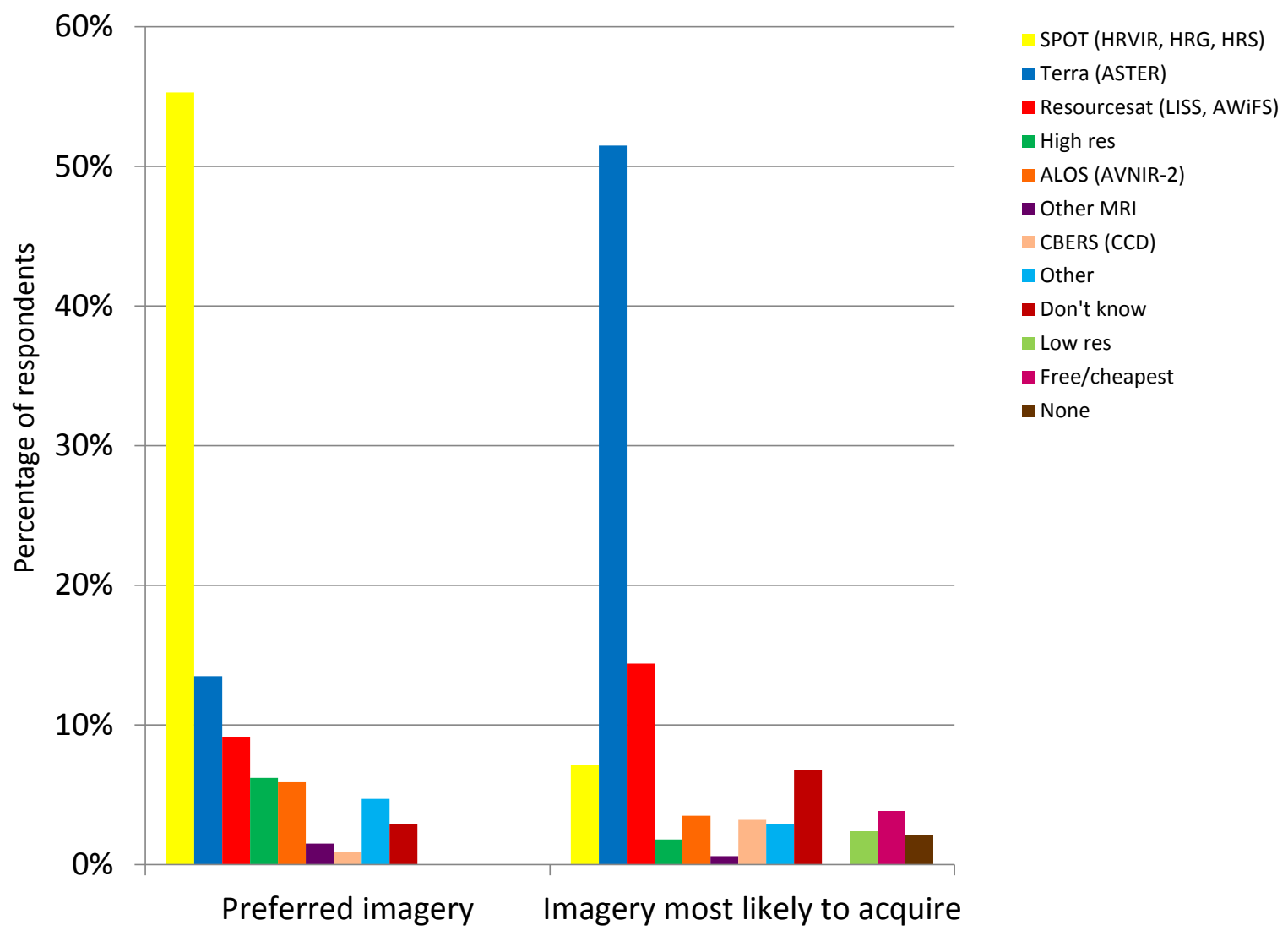

Figure 16. Preferred imagery versus imagery most likely to be acquired within budget constraints among current Landsat users who would substitute different imagery based on budget constraints if Landsat imagery was no longer available.

Another way to explore value is to examine what would happen to costs and revenues/funding if Landsat were no longer available. Increases in costs could occur, for instance, if users have to pay for other imagery, data, or field work to replace the information provided by Landsat imagery. Revenues could possibly decrease because a product based on Landsat can no longer be produced or the product must be created from a more expensive type of data. Typically, these sorts of budgetary questions can only be answered by certain individuals in an organization who have access to that information. We knew that not everyone in this sample would be able to respond to these questions; therefore, we only asked for information regarding the projects in which the respondents were involved. Respondents also had the option to indicate they did not know.

When asked to estimate the percentage increase in costs for their Landsat-related projects, almost half of the respondents felt their costs would increase by at least 1 percent, 41 percent didn't know if their costs would increase, and 11 percent felt their costs would not increase. Of those who believed their costs would increase $(n=668)$, the average total percentage increase in costs was 30 percent, which translated to about $\$ 27,000$ on average among those who were able to provide the current costs of all their projects which rely on 
Landsat imagery $(n=519)$. When asked about certain types of cost increases, more than half of the respondents said it is somewhat or very likely that total, processing, and

administration/overhead costs would increase and that more time would be spent on projects. However, most believed it is unlikely they would purchase additional equipment/software or hire more staff.

Regarding changes in revenues/funding, 43 percent of users did not know what impact the loss of Landsat would have. A third felt there would be no impact and one-fifth thought their revenues/funding would decrease.

\section{Willingness to Pay for Landsat Imagery}

Economic benefits, whether of a market or non-market good, are measured by the maximum amount the users would pay for another unit of it. For market goods, price measures the willingness to pay (WTP) for one more unit (for example, a pound of apples, a gallon of gas). Estimating the user benefits for non-market and publicly provided goods can be challenging because there is either no price (Landsat imagery is currently available at no cost) or only an administrative price (as was the case when Landsat imagery was provided for a fee). The administrative price often does not reflect market forces or an equilibrium price and quantity, and sometimes will substantially under or overstate what a user would pay. (A single administrative price will reveal a single point on the user's demand for the good.) Using WTP for both market and non-market goods ensures commensurability between dollar benefit estimates of these two types of goods.

WTP is the standard measure of benefits in benefit cost analysis (Sassone and Schaffer, 1978) and economists use a variety of techniques to estimate the WTP for non-market goods. Champ and others (2003) provide an accessible review of each of the commonly used methods. When there is no price, or there is little data available on what users will pay, a stated preference or intended behavior technique known as the Contingent Valuation Method (CVM) is commonly used. This method uses a simulated or hypothetical market to determine the maximum amount a user would pay for another unit of the good rather than use a more expensive substitute or do completely without. The method is recommended for use by federal agencies performing benefit cost analyses (Office of Management and Budget, 1992; U.S. Environmental Protection Agency, 2000; U.S. Water Resources Council, 1983). As suggested by the National Oceanic and Atmospheric Administration (NOAA) panel on contingent valuation (Arrow and others, 1993), we asked a dichotomous choice format question. In this case, the user was asked to decide only whether the Landsat scene is worth more than the cost specified in the question. The specific question asked:

"If Landsat 5 and 7 become inoperable before the next Landsat satellite is operational (scheduled to launch in 2012), you may have to obtain imagery elsewhere during the interim. Assume that you are restricted to your current project or agency budget level and that the money to pay this cost would have to come out of your existing budget. If such a break in continuity did occur and you had to pay for imagery that was equivalent to the Landsat standard product now available, would you pay \$XXX for one scene covering the area equivalent to a Landsat scene?”

The "\$XXX" was randomly filled in with one of 21 different dollar amounts. The dollar amounts ranged from a low of $\$ 5$ to a high of $\$ 5,000$. To measure an individual's maximum WTP based on Yes/No responses to a single dollar amount, it is important to provide a dollar 
amount low enough that nearly all Landsat users would answer Yes, and a dollar amount high enough that nearly all Landsat users would answer No. The remaining dollar amounts were $\$ 10$, $\$ 20, \$ 30, \$ 40, \$ 50, \$ 60, \$ 70, \$ 80, \$ 100, \$ 150, \$ 200, \$ 250, \$ 300, \$ 400, \$ 500, \$ 700, \$ 1,200$, 1,500 , and $\$ 2,500$. The response to this question provides the data necessary to calculate what is referred to as a "single-bounded WTP." The response to this question also allows the estimation and development of a demand function for the nonmarket good-as opposed to the single point on the demand function from administrative pricing. The demand function or curve is one of the outputs of CVM and is the combination of quantities consumed at different price amounts. This WTP question includes an explicit budget constraint ("Assume you are restricted to your current project or agency budget level...") and a reminder that the funds to pay the higher cost would have to come out of this fixed budget. This follows the recommendation of the NOAA panel (Arrow and others, 1993) that budget reminders are to be included in WTP questions.

Recognition of budget constraints is important to be consistent with consumer behavior and demand theory.

The demand curve derived from the responses to the WTP question is shown in figure 17. This demand curve can be used to determine the median and mean WTP for this sample of users. The median is $\$ 218$ per scene. This is not the value of the scene to the user but the value where a majority ( $>50$ percent) of the sampled users would purchase a scene equivalent to a Landsat scene. The mean value of a scene involves integrating the area under the curve in figure 17 . The curve in figure 17 is unbounded, meaning it does not have an upper limit. The value of Landsat imagery to some users is substantial and this pulls the average well above the median. The

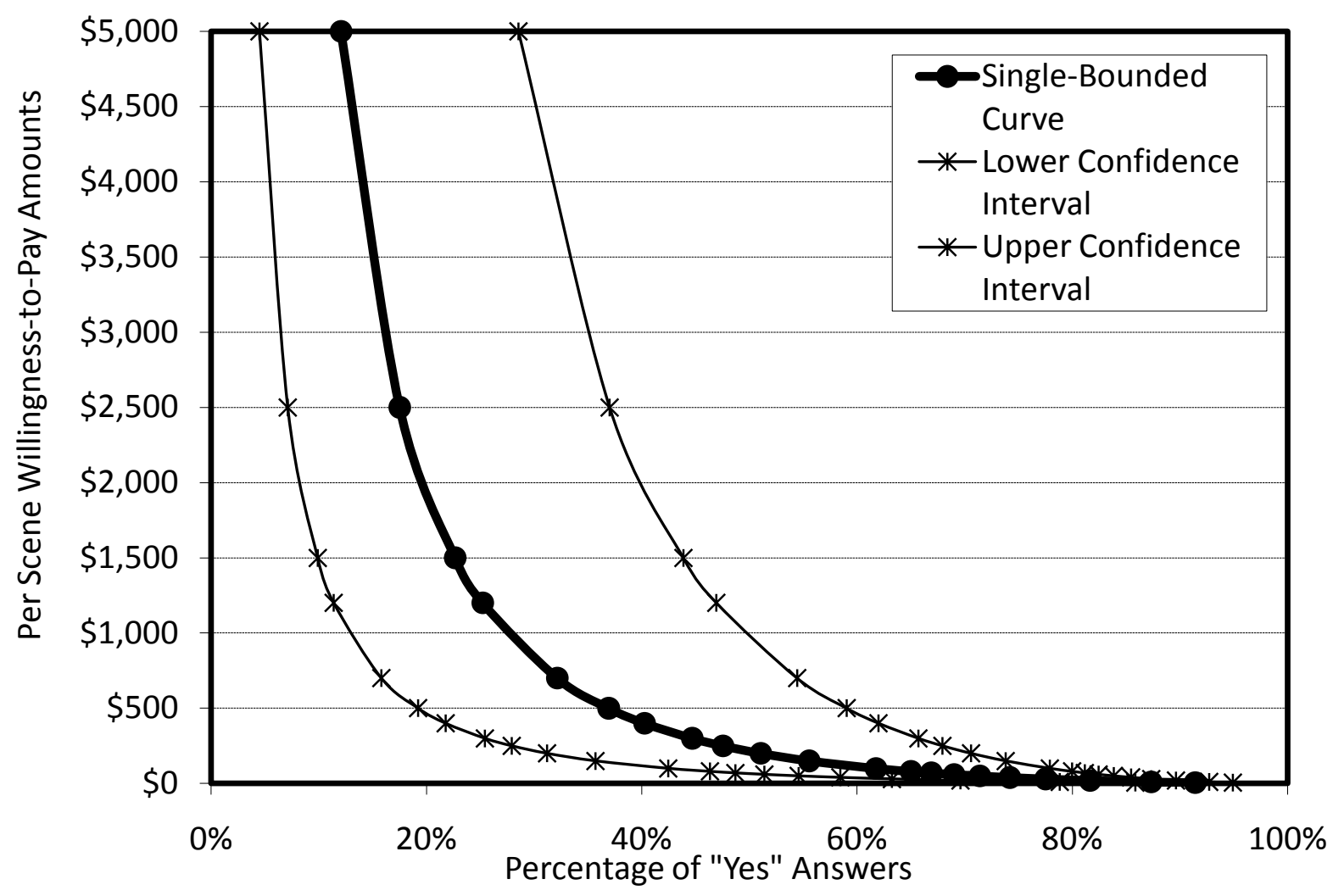

Figure 17. Single-bounded demand curve of willingness to pay for imagery to replace Landsat imagery among current Landsat users. 
standard practice when faced with an unbounded average is to use one of the high bids in the sample for the upper bound. To be conservative, we chose the second highest bid $(\$ 2,500)$ and calculated a mean WTP of $\$ 760$ per scene. The calculated mean WTP with an unbounded average is sensitive to the bid amount selected, choosing the highest bid $(\$ 5,000)$ would increase the mean and choosing the third highest bid $(\$ 1,500)$ would decrease it. Significant differences in mean WTP can be seen among sectors (fig. 18). Federal government users are willing to pay the most at more than $\$ 1,000$, followed by academic users at more than $\$ 800$, and private users at about $\$ 700$. Again, because the sample is not random, the reported WTP values should not be generalized to the population as a whole or any segments of the population.

The precision of the WTP estimate can be increased by asking respondents a follow-up dichotomous choice question based on the answer to the first question, thus collecting the data necessary for a double-bounded estimate of WTP (described in Appendix 3). In concept, the double-bounded analysis is intuitively appealing as statistical theory and past studies have shown that asking the second WTP question improves the statistical efficiency of WTP estimates. However, like other studies (for example, Cameron and Quiggin, 1994; Whitehead, 2002), we also found that the respondents' behavioral response to the second bid amount was somewhat different than the response to the first bid amount. Given this difference, we report the standard single-bounded CVM results described above because the method is best in terms of statistical significance of independent variables and goodness of fit (the percentage of correct predictions is about 70 percent) and is a well-accepted industry standard.

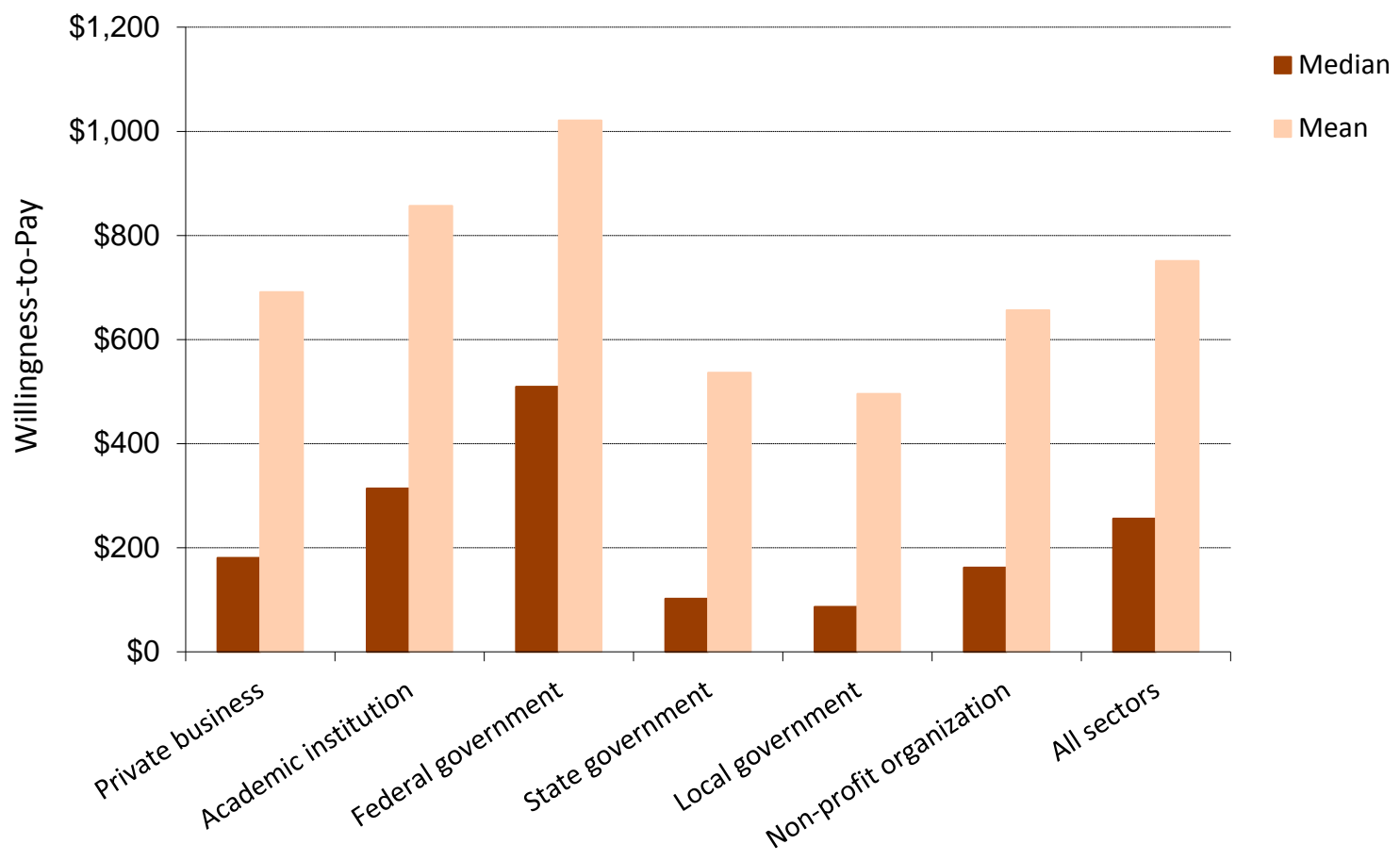

Figure 18. Single-bounded logit model results for willingness to pay for imagery to replace Landsat imagery among current Landsat users. 
The value of Landsat imagery was high overall for these respondents, and those in certain sectors, such as academia, found it even more valuable than respondents in other sectors. In general, Landsat imagery was important to respondents for their work and they were very satisfied with the attributes provided by Landsat. They found it beneficial for improving decisionmaking and preventing harm to the environment and humans, among many other benefits. These benefits will likely increase as the no-cost policy change becomes widely known, LDCM is launched, and emerging issues facing the nation, such as climate change, become more pronounced and require increasing amounts of global, reliable data. The value of Landsat imagery to these respondents is also demonstrated by the substantial amount of work that would be discontinued or require a substitute source of data. Finally, the value is demonstrated by respondents' willingness to pay for the good. On average, respondents were willing to pay $\$ 760$ per scene, which is greater than the previous administratively set price.

\section{Conclusion}

The results of the survey revealed that respondents from multiple sectors use Landsat imagery in many different ways, as demonstrated by the breadth of project locations and scales, as well as application areas. The current level of use will likely increase among these respondents, particularly as it becomes better known that the imagery is available at no cost and as new uses are identified. The changes in acquisition patterns, including the increase in the number of scenes acquired and the decreasing amount spent after the imagery became available at no cost also point toward increases in future use.

The value of Landsat imagery to these users was demonstrated by the high importance placed on the imagery, the numerous benefits received from projects using Landsat imagery, the negative impacts if Landsat imagery was no longer available, and the substantial willingness to pay for replacement imagery in the event of a data gap. These results represent only a minimum value of Landsat within the Landsat user community; further research would help to determine what the value of the imagery is to a greater segment of the population.

Throughout the analyses, differences between sectors occurred on almost all variables. The identification of the "local users" group helped to explain some of these differences, but respondents in the academic and federal government sectors also tended to differ from the rest of the respondents. They tended to be more technical users, used the imagery more heavily, and were willing to pay more for replacement imagery. These differences between sectors merit further exploration in future studies to see whether they exist within the other segments of the population as well.

The survey collected information from users who are both part of and apart from the known user community. The diversity of the sample delivered results that provide a baseline of knowledge about the users, uses, and value of Landsat imagery. While the results supply a wealth of information on their own, they can also be built upon through further research to generate a more complete picture of the population of Landsat users as a whole. Surveying other segments of the Landsat user population, such as those who procure imagery from EROS and international users, and conducting case studies on the value of Landsat imagery in specific applications would provide additional useful information. 


\section{Acknowledgments}

This study was funded by the U.S. Geological Survey’s (USGS) Land Remote Sensing Program and is part of a larger study being conducted in conjunction with the USGS Western Geographic Science Center (WGSC) in Menlo Park, Calif. We would like to thank: Catherine Lundy from Eagle River Water and Sanitation District (Vail, Colo.) for her initial analysis of qualitative data from the survey while a student at Colorado State University; Eric Wood, Tom Loveland, and John Dwyer from the USGS Earth Resources Observation and Science (EROS) Center in Sioux Falls, S. Dak., for their invaluable help in constructing, reviewing and testing the survey instrument; Katie Walters (USGS Fort Collins Science Center), Makiko Hong and Tamara Wilson (WGSC) for their work during the sampling phase of the project; survey pre-test participants from EROS and South Dakota State University; and the numerous people who reviewed the survey and provided constructive feedback, including Richard Bernkopf (WGSC), Molly Macauley (Resources for the Future), participants in our session at the $17^{\text {th }}$ William T. Pecora Memorial Remote Sensing Symposium, and members of the Landsat Science Team. 


\section{References}

American Society for Photogrammetry and Remote Sensing, 2006, Survey on the future of land imaging: Bethesda, Md., American Society for Photogrammetry and Remote Sensing.

Arrow, Kenneth, Solow, Robert, Portney, P.R., Leamer, E.E., Radner, Roy, and Schuman, Howard, 1993, Report of the NOAA panel on contingent valuation: Wash., D.C., National Oceanic and Atmospheric Administration, U.S. Department of Commerce.

Atkinson, Rowland, and Flint, John, 2001, Accessing hidden and hard-to-reach populationsSnowball research strategies: University of Surrey Social Research Update, v. 33. (Accessed on January 2, 2007, at http://sru.soc.surrey.ac.uk/SRU33.html.)

Avico, U., Kaplan, C.D., Korczak, D. and van Meter, K.M., 1988, Cocaine epidemiology in three European Community cities_-A pilot study using a snowball sampling methodology:

Rotterdam, Netherlands, Insituut voor Verslavingsonderzoek.

Bachman, Ronet, and Schutt, R.K., 2007, The practice of research in criminology and criminal justice ( $3^{\text {rd }}$ edition), Thousand Oaks, Calif., Sage Publications.

Berg, Sven, 1988, Snowball sampling, in Kotz, Samuel, and Johnson, N.L., eds., Encyclopedia of Statistical Sciences v. 8, p. 528-532.

Biernacki, Patrick, and Waldorf. D., 1981, Snowball sampling-Problems and techniques of chain referral sampling: Sociological Methods Research, v. 10, p. 141-163.

Blanken, P.B., Hendricks, V.M., and Adriaans, N.F.P., 1992, Snowball samplingMethodological analysis?, in Hendricks, V.M., Blanken, P.B., and Adriaans, N.F.P., eds., Snowball sampling-A pilot study on cocaine use: Rotterdam, Netherlands, Insituut voor Verslavingsonderzoek, p. 83-100.

Browne, Kath, 2005, Snowball sampling-Using social networks to research non-heterosexual women: International Journal of Social Research Methodology, v. 8, no. 1, p. 47-60.

Cameron, T.A., and Quiggin, J.C., 1994, Estimation using contingent valuation data from a dichotomous choice with follow-up questionnaire: Journal of Environmental Economics, v. 27L, p. 218-234.

Champ, P.A., Boyle, K.J., and Brown, T.C., eds., 2003, A primer on nonmarket valuation (The economics of non-market goods and resources series, Bateman, I.J., ed.): Norwell, Mass., Kluwer Academic Publishers, 577 p.

Cohen, Jacob, 1988, Statistical power and analysis for the behavioral sciences ( $\left.2^{\text {nd }} e d.\right)$, Hillsdale, N.J., Lawrence Erlbaum Associates, Inc.

Coleman, J.S., 1958, Snowball sampling-Problems and techniques of chain referral sampling: Human Organization, v. 17, p. 28-36.

Dillman, D.A., 2007, Mail and Internet surveys-The tailored design method, $2^{\text {nd }}$ edition: Hoboken, N.J., John Wiley \& Sons, Inc., 523 p.

EarthSat Corp., 1974, Executive summary-Earthsat study on the value of ERTS information.

ECON, Inc., 1974, The economic value of remote sensing of earth resources from space-An ERTS overview and the value of continuity of service: Princeton, N.J., ECON, Inc., 134 p. (Available at: http://ntrs.nasa.gov/archive/nasa/casi.ntrs.nasa.gov/19750006142_1975006142.pdf)

Eland-Goossenson, M.A., Van de Goor, L.A.M., and Vollemans, E.C., 1997, Snowball sampling applied to opiate addicts outside the treatment system: Addiction Research, v. 5, p. 317-330. EROS (Earth Resources Observation Science Center), 2007, Customer satisfaction surveyLandsat data: Sioux Falls, S. Dak., Earth Resources Observation Science Center, 78 p. 
Faugier, Jean, and Sargeant, Mary, 1997, Sampling hard to reach populations: Journal of Advanced Nursing, v. 26, p. 790-797.

Fisher, Nigel, Turner, S.W., Pugh, Robert, and Taylor, Colin, 1994, Estimating the number of homeless and homeless mentally ill people in north east Westminster by using capturerecapture analysis: British Medical Journal, v. 308, p. 27-30.

FLIIWG (Future of Land Imaging Interagency Working Group), 2007, A plan for a U.S. national land imaging program: Wash., D.C., Office of Science and Technology Policy and National Science and Technology Council, 120 p. (Available at: http://www.landimaging.gov/fli_iwg_report_print_ready_low_res.pdf.)

Goodman, L.A., 1961, Snowball sampling: The Annals of Mathematical Statistics, v. 32, no. 1, p. 148-170.

Griffiths, Paul, Gossop, Michael, Powis, Beverly, and Strang, John, 1993, Reaching hidden populations of drug users by privileged access interviewers-Methodological and practical issues: Addiction, v. 88, p. 1617-1626.

Heckathorn, D.D., 2002, Respondent-driven sampling II—Deriving valid population estimates from chain-referral samples of hidden populations: Social Problems, v. 49, p. 11-34.

Henslin, J.M, 1972, Studying deviance in four settings-Research experiences with cabbies, suicide, drug users, and abortionees, in Douglas, J.D., ed., Research on deviance: New York, Random House, p. 35-70.

Kaplan, C.D., Korf, Dirk, and Sterk, Claire, 1987, Temporal and social contexts of heroin-using populations-An illustration of the snowball sampling technique: Journal of Mental and Nervous Disorders, v. 175, no. 9, p. 566-574.

Lozar Manfreda, Katja, Bosnjak, Michael, Berzelak, Jernej, Haas, Iris, and Vehovar, Vasja, 2008, Web surveys versus other survey modes - A meta-analysis comparing response rates: International Journal of Market Research, v. 50, no. 1, p. 79-104.

Macauley, M.K., 2005, The value of information-A background paper on measuring the contribution of space-derived earth science data to resource management (Discussion paper 05-26): Wash, D.C., Resources for the Future, 27 p.

Macauley, M.K., 2006, The value of information-Measuring the contribution of space-derived earth science data to resource management: Space Policy, v. 22, p. 274-282.

Martilla, J.A., and James, J.C., 1977, Importance-performance analysis: Journal of Marketing, v. 2, no. 1, p. 77-79.

Murphy, K.R., and Myors, Brett, 1998, Statistical power analysis-A simple and general model for traditional and modern hypothesis tests: Mahwah, N.J., Lawrence Erlbaum Associates, Inc. National States Geographic Information Council, 2006, Results of a national survey-Imagery for the Nation Proposal: Bel Air, Md., National States Geographic Information Council, 40 p.

Office of Management and Budget, 1992, Guidelines and discount rates for benefit-cost analysis of federal programs, Circular A-94, Revised Transmittal Memo \#64: Wash., D.C., Executive Office of the President.

Roberts, Sam, 2007, In challenge to survey low-wage workers, researchers pursue 'snowball sample’: New York Times, April 15.

Sassone, P.G., and Schaffer, W.A., 1978, Cost-benefit analysis-A handbook: New York, N.Y., Academic Press, 190 p.

Sheehan, K.B., 2001, E-mail survey response rates-A review: Journal of Computer-Mediated Communication, v. 6, no. 2. (Also available at http://jcmc.indiana.edu/vol6/issue2/ sheehan.html.) 
Sheu, S.J., Wei, I.L, Chen, C.H., Yu, Shu, and Tan, F.I., 2009, Using snowball sampling method with nurses to understand medication administration errors: Journal of Clinical Nursing, v. 18, no. 4, p. 559-569.

Shih, T.H., and Fan, Xitao, 2008, Comparing response rates from web and mail surveys-A meta-analysis: Field Methods, v. 20, no. 3, p. 249-271.

Spreen, Marinus, 1992, Rare populations, hidden populations and link-tracing designs-What and why?: Bulletin Methodologie Sociologique, v. 36, p. 34-58.

Stoney, W., Fletcher, A., and Lowe, A., 2001, Data only report of Landsat user survey (draft): Falls Church, Va., Mitretek Systems, 85 p.

Sudman, Seymour, and Freeman, H.E., 1988, The use of network sampling for locating the seriously ill: Medical Care, v. 26, no. 10, p. 992-999.

Tsvetovat, Max, and Sharabati, Walid, 2006, CSS 692-Social Network Analysis: LiveJournal, Fall, p. 1-20. (Accessed on January 4, 2007, at http://www.academic2.american.edu/ sharabat/files/SNA_Problem_Set2.pdf, available on January 19, 2010 at: http://web.ics.purdue.edu/ wsharaba/files/SNA_Problem_Set2.pdf.)

U.S. Environmental Protection Agency, 2000, Guidelines for preparing economic analyses, EPA 240-R-00-003: Wash., D.C., U.S. Environmental Protection Agency.

U.S. Water Resources Council, 1983, Economic and environmental principles and guidelines for water and related land resources implementation studies: Wash, D.C., U.S. Government Printing Office.

Van Meter, K.M., 1990, Methodological and design issues-Techniques for assessing the representatives of snowball samples, in Lambert, E.Y., ed., The collection and interpretation of data from hidden populations: Rockville, Md., National Institute on Drug Abuse, NIDA Research Monograph 98, p. 31-43.

Vogt, W.P., 1999, Dictionary of statistics and methodology—A nontechnical guide for the social sciences: London, Sage Publications.

Watters, J.K., and Biernacki, Patrick, 1989, Targeted sampling-Options for the study of hidden populations: Social Problems, v. 36, p. 416-430.

Wejnert, Cyprian, and Heckathorn, D.D., 2008, Web-based network sampling-Efficiency and efficacy of respondent-driven sampling for online research: Sociological Methods and Research, v. 37, no. 1, p. 105-134.

Whitehead, J.C., 2002, Incentive incompatibility and starting point bias in iterative valuation questions: Land Economics, v. 78, no. 2, p. 285-297. 


\section{Appendixes}

\section{Appendix 1}

\section{Snowball Sampling}

When the population under study is unknown or hidden, traditional sampling methodologies cannot be applied. Because this type of population is difficult to locate and access, a random selection of the population (probability sampling) cannot occur (Heckathorn, 2002). Instead, more purposive methodologies such as snowball sampling must be employed to make use of the social networks that exist between known members of the population (Coleman, 1958; Goodman, 1961; Spreen, 1992). Snowball sampling has long been used for reaching populations that are hidden and vulnerable, such as drug users, prostitutes, criminals, the mentally ill and the homeless (for example, Avico and others, 1988; Bachman and Schutt, 2007; Eland-Goossenson and others, 1997; Faugier and Sargeant, 1997; Fisher and others, 1994; Griffiths and others, 1993; Kaplan and others, 1987; Sudman and Freeman, 1988; Waters and Biernacki, 1989). Typically, hidden and vulnerable populations can only be accessed once the individual enters an institutional setting such as a prison, drug rehabilitation center, or homeless shelter. However, these individuals are a captive population and do not necessarily represent the entire population (Waters and Biernacki, 1989). More recently, snowball sampling has also been used to research populations that are not hidden, but may be difficult to reach through traditional sampling methods, such as nurses (Sheu and others, 2009) and musicians and artists (Roberts, 2007). There is no comprehensive list of members of these types of populations to sample from and, though they occur as part of the general population, they are often too rare to be reached via a survey of the general public. A primary advantage of snowball sampling is that it is very successful at accessing individuals in hidden and (or) hard-to-reach populations. Results can be produced quickly and cost-effectively. It has been noted that web-based snowball sampling can achieve results up to twenty times faster than traditional methods such as face-to-face interviews and mail-based referrals (Wejnert and Heckathorn, 2008). Another advantage is that snowballing allows researchers to trace the networks in the target population, leading to a better understanding of how social networks and communication operate in the population.

\section{Snowball Sampling Process}

The snowball sampling process is relatively simple. The links that exist between individuals in the target population allow "a series of referrals to be made within a circle of acquaintances” (Berg, 1988). The initial step involves identifying a group of individuals who are known members of the population to create a seed (Vogt, 1999). For hidden populations, this may involve contacting people who interact with population members on a regular basis. For instance, contacting the staff at an AIDS clinic or a drug rehabilitation center would be one way to reach people with AIDS or drug addictions. For less sensitive populations, initial contacts may be conducted through professional organizations or other known groups. With some populations, a web search based on appropriate keywords for potential members of the populations can be effective. After the seed is established, those individuals are contacted to elicit the names of other individuals in the target population. This produces the first wave of individuals, who, in turn, are contacted, thus providing the second wave. The outcome from contacting the second wave produces the third wave which contributes to the fourth wave and so on. There are a number of variables to consider when designing snowball sampling. First, the number of wave 
iterations may or may not be determined a priori. Often, the process ends when the waves cease to produce a predetermined number of new contacts. In small populations, it may take only a few waves before almost no new contacts are obtained in a wave. Second, the number of contacts a participant is requested to provide can vary. Typically, the number of contacts asked for is three, partly to minimize the burden on the respondent but also to ensure that the recommended people are truly part of the population under study. In certain cases, such as particularly stigmatized or rare populations, participants are asked for an exhaustive list of contacts. Third, the criteria for including a participant in the sample can also vary. Usually, the number of times a person is identified by others as a member of a certain population is used. For some populations, it is appropriate to include a participant after only one mention, while for others, it may require two or three mentions.

\section{Methodological Biases}

There are several methodological biases in snowball sampling (Heckathorn, 2002). The initial sample or seed may introduce immediate bias because the people who comprise the seed are typically selected via a convenience sample. Volunteerism bias also frequently exists, both in the seed and also in subsequent waves. Masking is also common, particularly among sensitive populations. People may not want to reveal that their friends and acquaintances are members of the population. Another bias, typically referred to as homophily bias, also often exists in snowball samples. Because the social network of an individual tends to include people who are similar in many respects, such as age, education, and race and ethnicity, the people they refer will reflect those similarities. However, this may not be the case in populations where members are not linked personally, but by the work they do, for instance. Finally, the number of people an individual knows, which can range from single digits into the hundreds, influences the chances a person will be included in the sample. Members of the population with the largest personal networks and highest 'social visibility' are more likely to be referred (Biernacki and Waldorf, 1981; Henslin, 1972). Conversely, those with small networks, or isolated individuals, can be omitted from the sample (Van Meter, 1990).

These biases limit the generalizability of data gathered from a snowball sample and make it very difficult to determine the size of the population and the statistical accuracy of samples. There are some statistical methods specific to unknown population sizes that can be used for population estimates, but typically results from a snowball sample are not considered to be generalizable to the population at large (Atkinson and Flint, 2001; Tsvetovat and Sharabati, 2006).

Many of these biases may be mitigated by taking a few extra steps. Though snowball sampling results in a non-random sample, the sample approaches randomness as more and more individuals in the population are contacted, thus reducing biases (Atkinson and Flint, 2001; Tsvetovat \& Sharabati, 2006). Additionally, by relying on a variety of indirect sources, such as a web search, to develop a seed from an unknown population, researchers minimize the potential for bias (Faugier and Sargeant, 1997; Blanken and others, 1992). Sometimes, for populations about which little is known, snowball sampling can provide an important means to obtain information about members of a population that might not otherwise be available. 


\section{Appendix 2}

Users of Other Types of Satellite Imagery

As part of the U.S. Geological Survey's effort to survey Landsat users, users of other types of satellite imagery were also contacted. In addition to Landsat users, there were three main groups of users who were also surveyed:

1. Current users of other types of MRI (not Landsat)

2. Past users of Landsat imagery

3. Non-MRI users (for example, users of high or low-resolution imagery)

The main objective in asking questions of these groups was to discover why they were not currently using Landsat, as well as to discover any differences between these groups and the current Landsat users.

\section{Current Users of Other Types of MRI}

The survey was constructed to allow direct comparisons between current Landsat users $(n=1,392)$ and current users of other MRI $(n=167)$. However, the results suggest that at least some of those who indicated they were not currently using Landsat were, in fact, using it but did not know. Because respondents in this group could very well have answered the questions in reference to Landsat imagery without realizing it, the responses from this group may not be an accurate reflection of their use of MRI other than Landsat. Given the uncertain nature of the users in this group, as well as the relatively small number of respondents in the group, results from this group are not reported.

\section{Past Landsat Imagery Users}

This group consisted of respondents who had used Landsat at some point in the past but had not used it in the year previous to the survey $(n=445)$. Of those users, 20 percent were currently using other types of MRI and 80 percent were not. When asked why they were not using Landsat, almost 70 percent of respondents stated that their work did not require the imagery. Many respondents also cited spatial resolution not meeting needs (43 percent), other sources of imagery being more attractive (33 percent), and insufficient data quality (22 percent) as main reasons. When asked if they would use Landsat imagery again in the future, 60 percent of the past Landsat users in our sample said they would. People who were not currently using MRI of any kind ( $n=356$ ) were also asked what would make them more likely to use MRI in the future. Just over 70 percent selected changes in their work, 53 percent cited improved spatial resolution, and 34 percent chose decreases in cost. Many respondents also cited better accessibility (29 percent), improved temporal resolution (29 percent), improved spectral resolution (26 percent) and better availability (26 percent).

For the most part, the past Landsat users in the sample were very similar demographically to the current Landsat users. About 75 percent of each group was male, more than 90 percent were white, the average age was in the late forties, and well over half held a master's or doctoral degree. However, there were some differences in education and sector between past and current Landsat users. Though both groups were highly educated, only 17 percent of past Landsat users had a Ph.D., compared to 32 percent of current Landsat users. Additionally, almost 24 percent of past Landsat users had a bachelor's degree compared to 17 percent of current Landsat users. These dissimilarities may be the result of differences in the sector distribution among each group. The primary sectors among past Landsat users were local government (25 percent), 
private business (20 percent), and state government (19 percent). This differs from the sector breakdown among current Landsat users in the sample, which was dominated by academia (33 percent), followed by private business (18 percent), and federal government (17 percent).

\section{Non-MRI Users}

This group consisted of respondents who had never used MRI, but who had used high- or low-resolution imagery at some point for their work $(n=229)$. Of these users, about 64 percent had used only high-resolution imagery, just over 31 percent had used only low-resolution imagery, and 4 percent had used both types of imagery. The main reasons for not using MRI were similar to those of past Landsat users. Almost 61 percent of the non-MRI users in the sample said their work has not required the imagery, 38 percent stated the spatial resolution had not met their needs, and 31 percent cited insufficient data quality. More than half of the respondents in this group said they did not foresee using MRI in the future. When asked what would make them more likely to use MRI, the most common responses were changes in work (57 percent of respondents), improved spatial resolution (31 percent), better accessibility (26 percent), decrease in cost ( 25 percent), and better availability (25 percent). Almost 12 percent of respondents said nothing would make them more likely to use MRI in the future.

For the most part, the non-MRI users in the sample were very similar demographically to both the current and past Landsat users. About 75 percent or more of each group was male, more than 90 percent were white, and the average age was in the late forties. However, there were some differences between current Landsat users and non-MRI users. As was the case with past Landsat users, both groups were highly educated, but only 11 percent of non-MRI users had a Ph.D., compared to 32 percent of current Landsat users. Additionally, far more non-MRI users held bachelor's degrees (33 percent) than either current (17 percent) or past (24 percent) Landsat users. Again, these dissimilarities may be the result of differences in the sector distribution among each group. The primary sectors among non-MRI users were local government (30 percent), private business (28 percent), and state government (17 percent). This is similar to the distribution of sectors among past Landsat users, but differs from the sector breakdown among current Landsat users in the sample, which was dominated by academia (33 percent), followed by private business (18 percent) and federal government (17 percent).

\section{Discussion}

The results from past Landsat users and non-MRI users in this sample indicate that the use of MRI, and specifically Landsat, depended greatly on the work respondents were doing. However, the spatial resolution of MRI was also an impediment, as was insufficient data quality. Because respondents' work is out of the control of data providers and the spatial resolution of most MRI, including Landsat, is unlikely to change in the near future, these barriers to using MRI are not likely to be removed. However, there were some respondents in each group who believed that better accessibility, better availability, and lower cost of MRI would make them more likely to use it. These types of barriers could be addressed by data providers if desired, as was done by providing Landsat at no cost. Future research could investigate whether no cost Landsat imagery has resulted in non-Landsat users either returning to, or using Landsat for the first time.

These past Landsat users and non-MRI users were demographically similar to current Landsat users in regards to gender, race and ethnicity, and age. However, the results indicate that Landsat users might have different educational backgrounds and work in different sectors than 
users of other types of satellite imagery. Because the sample taken for this study is not random, more research is needed to determine if these differences are also present at the population level or exist within just this sample of users. 


\section{Appendix 3}

\section{Double-Bounded Willingness-To-Pay Analysis}

The statistical efficiency of the WTP estimate can be increased by asking respondents a second dichotomous choice question, thus collecting the data necessary for a double-bounded estimate of WTP. With the first WTP response, we know that the respondent's WTP is higher or lower than the dollar amount asked to pay. If those who answer Yes to the first question are asked if they would pay a higher amount, we can obtain additional information about their WTP: (a) if they state YES to the second, higher dollar amount, we know their WTP is even higher than the first bid amount and in fact is higher than the increased bid amount (the second dollar amount is twice the first in this case); (b) if they state they would not pay this second higher dollar amount we know their WTP is bounded between the first and second dollar amount, thus bracketing their maximum WTP between the first and second dollar amount. The same pattern holds for those answering NO to the first dollar amount. If a respondent answers YES to the second dollar amount (half the first dollar amount in this case), we can potentially bracket their WTP between the first and second dollar amount. This second question can substantially improve the efficiency of estimating the WTP but risks offending the respondent with a moving price target.

After the first and second WTP questions, respondents were asked how certain they are that they would (or would not) pay that price on a scale of 0 to 100 percent. This provides further information about the actual WTP of each respondent and allows for examination of the quality of each respondent's answers. For example, if a respondent says YES to the first WTP amount and YES to the second higher amount, economic logic suggests they should be less certain (or the same) about paying the higher amount.

In concept, the double-bounded analysis is intuitively appealing. However, like other studies have found (for example, Cameron and Quiggin, 1994; Whitehead, 2002), we also found that the respondents' behavioral response to the second bid amount is somewhat different than the response to the first bid amount. For example, one respondent said No to $\$ 150$ with a certainty of 40 percent, but then said No to $\$ 75$ with a certainty of 60 percent. In practice, it appears that respondents do not like the follow-up question with the different bid amount. The information gathered from the second question may not be as good as the information from the first question. For both the first and second questions, the probability of saying Yes goes down as the bid amount increases, but at somewhat different rates in response to each question. There are several reasons this may be the case, including strategic behavior on the part of respondents, uncooperative behavior, or changed preferences. At this time, we hypothesize that the behavioral shift between first and second responses may be due to the large magnitude of the increase between the first and second bids. Especially at high first dollar amounts $(\$ 1,500, \$ 2,500$, and $\$ 5,000)$, the very large absolute magnitude of the increase in the second dollar amount $(\$ 3,000$, $\$ 5,000$ and $\$ 10,000$ ) may have reduced the credibility of the second WTP question to the respondent. It is possible that the response to this second question was more of a signal about the question itself rather than a second "snapshot" of their WTP.

The demand curve of the double-bounded analysis does behave as expected when compared to the curve of the single-bounded analysis (fig. 19). The double-bounded curve shifts to the left and has much tighter confidence intervals than the single-bounded curve. But there are no significant differences found between sectors in the double-bounded analysis. The reasonable differences in WTP across groups of respondents disappear with the information from the second 


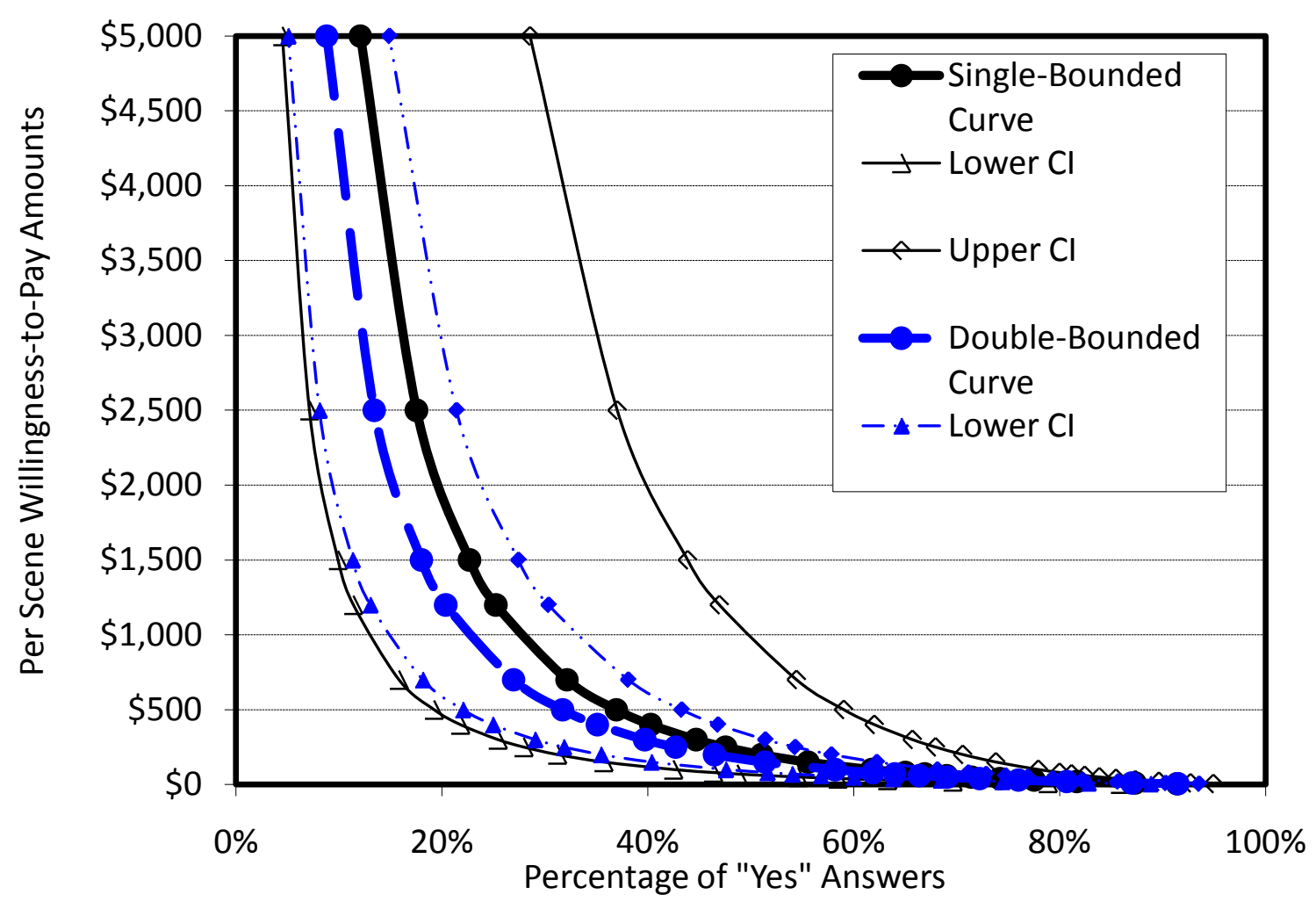

Figure 19. Single- and double-bounded demand curves of willingness to pay for imagery to replace Landsat.

question. The standard errors on the sector variables were larger than the parameter estimates so the variables can reasonably be removed from the double-bounded model.

Single-Bounded vs. Double-Bounded WTP Analyses

The double-bounded analysis improves efficiency but the second question does not have as much value as the first question due to the behavioral shift between questions. This behavior was not expected among Landsat users, since they have experience with the imagery and know the good being valued quite well in most cases. Given the results, we chose to report the standard single-bounded dichotomous choice CVM results because they are the best in terms of the statistical significance of independent variables, goodness of fit (the percentage of correct predictions is about 70 percent), and the method is a well-accepted industry standard.

Several things might be tried for a future WTP for Landsat imagery question to reduce the behavioral difference between the first and second questions. The magnitude of the change between the first and second bid could be reduced from twice and half to 1.25 and 0.75 . The number of initial high bid amounts could be increased since these higher amounts are essential to accurate and precise estimates of mean WTP, which involves integration across the entire demand function. More information about respondents' WTP at these high bids would allow better estimation of the complete demand function. Other statistical modeling techniques such as ordered logit models that allow for one category for each of the four combinations of responses (Yes-Yes, Yes-No, No-Yes, and No-No) could also be tried. 
Publishing support provided by:

Denver Publishing Service Center

For more information concerning this publication, contact: Center Director, USGS Fort Collins Science Center

2150 Centre Ave., Bldg. C

Fort Collins, CO 80526-8118

(970) 226-9398

Or visit the Fort Collins Science Center Web site at: http://www.fort.usgs.gov/ 